\title{
Low Friction and Wear Resistant Carbon Nitride Thin Films for Rolling Components Grown by Magnetron Sputtering
}

\author{
Konstantinos D. Bakoglidis
}

\section{Linköping University INSTITUTE OF TECHNOLOGY}

Thin Film Physics Division

Department of Physics, Chemistry and Biology (IFM)

Linköping University

SE-581 83 Linköping, Sweden

Linköping 2015 
(C)Konstantinos D. Bakoglidis 2015

ISBN: 978-91-7519-051-8

ISSN: 0280-7971

Printed by LiU-tryck

Linköping, Sweden, 2015 


\title{
Low Friction and Wear Resistant Carbon Nitride Thin Films for Rolling Components Grown by Magnetron Sputtering
}

\author{
Konstantinos D. Bakoglidis
}

\begin{abstract}
The scope of this licentiate thesis is the investigation of carbon based thin films suitable for rolling components, especially roller bearings. Carbon and carbon nitride are materials with advantageous tribological properties and high resiliency. Such materials are required in order to withstand the demanding conditions of bearing operation, such as high loads and corrosive environments. A fundamental condition for coated bearings is that the deposition temperature must be striktly limited. Thus, carbon nitride $\left(C N_{x}\right)$ thin films were synthesized here at low temperature of $150^{\circ} \mathrm{C}$ by different reactive magnetron sputtering techniques, which are mid-frequency magnetron sputtering (MFMS), direct current magnetron sputtering (DCMS), and high power impulse magnetron sputtering (HiPIMS). While DCMS is a very well studied technique for carbon based films, MFMS and HiPIMS are relatively new sputtering techniques for carbon, and especially $C N_{x}$ depositions. Using different magnetron sputtering techniques, different ionization conditions prevail in the chamber during each process and influence the obtained film properties at a great extent. It was found that bias duty cycles and the amount of working gas ions are key parameters and affect the morphology and microstructure as well as the mechanical response of the films. Moreover, different bias voltages, from $20 \mathrm{~V}$ up to $120 \mathrm{~V}$ were applied during the processes in order to investigate the changes that the different ion energies induce in the film structure.

The structural, mechanical and tribological properties of $C N_{x}$ films are also presented in this licentiate thesis. The morphology of $C N_{x}$ films strongly depends on both the deposition technique and ion energy. The special configuration of MFMS mode produces highly homogeneous and dense films even from low applied bias voltages, while in HiPIMS mode high bias voltages above $100 \mathrm{~V}$ must be applied in order to produce films with similar structural characteristics. DCMS is also proven as a good technique for homogeneous and dense films. Low bias voltages do not favor
\end{abstract}


homogeneous structures, thus at $20 \mathrm{~V}$ all techniques produced films with columnar structures with intercolumnar voids. High bias voltages influence the $\mathrm{N}$ incorporation in the films, with the appearance of re-sputtering of $N$-containing species and a promotion of $s p^{2}$ bonding configurations with increasing ion energy. Nevertheless, the different deposition mode influences the $s p^{2}$ content in different ways, with only MFMS showing a clear increase of $s p^{2}$ content with increasing bias voltage and HiPIMS showing relatively constant $s p^{2}$ content. The morphology and microstructure of the $C N_{x}$ films affects their mechanical response, with higher ion energies producing harder films. A dependency of hardness and elastic modulus with increasing ion energy was obtained, where for all deposition modes, hardness and elastic modulus increase linearly with increasing bias voltage. Films with hardness as high as $\sim 25$ $G P a$ were synthesized by MFMS at $120 \mathrm{~V}$, while the softer film yielded a hardness of $\sim 7 \mathrm{GPa}$ and was deposited by HiPIMS at $20 \mathrm{~V}$. The elastic recovery of the films differs with increasing ion energies, presenting a correlation with the $\mathrm{C} s p^{2}$ bond content. The highest elastic recovery of $90 \%$ was extracted for the film deposited by MFMS at $120 \mathrm{~V}$ and is a value similar to the elastic recovery obtained for $F L$ $C N_{x}$ films. All films developed compressive residual stresses, depending also on the ion energies and the deposition mode used. It is demonstrated that the induced stresses in the films increase when denser and more homogeneous film morphologies are obtained and with higher $A r$ intercalcation. Low friction coefficients were obtained for all films between 0.05 and 0.07 , although the deposition conditions are not detrimental for the development of friction coefficient. The wear resistance of the films was found to be dependent on the morphology and to some extent on the microstructure of the films. Harder, denser, and more homogeneous films have higher wear resistance. Especially, $C N_{x}$ films deposited by MFMS at $120 \mathrm{~V}$ present no wear.

The tribological characteristics of the surface of the films were also investigated at nanoscale by a new reciprocal wear test. In this wear test, the recording of the track profile is performed in between consecutive test cycles, eliminating also thermal drift. The very low wear of the films deposited by MFMS at $100 \mathrm{~V}$ and $120 \mathrm{~V}$ revealed that during the wear test a phase transformation on the surface may take place, possibly graphitization. It is also demonstrated the way that the surface characteristics, such as asperities and roughness affects the tribological measurements. Attention is also turned to the presence of large asperities on the film surface and the way they affect the obtained average friction coefficient and tribological measured data. 


\section{Acknowledgements}

For the completion of this licentiate thesis, I would like to thank:

Lars Hultman, my main supervisor. Thank you for the trust in me, it gives me strength and patience to continue and carry out all the work. Thank you also for the help you provide and the useful intellectual discussions, despite your two jobs.

Grzegorz Greczynski, my co-supervisor. Thank you for acting as main supervisor, your valuable time and all our scientific discussions.

Susann Schmidt and Esteban Broitman, my other co-supervisors. Thanks for training me in the lab, for your time and your valuable responses to the problems we encounter. Your help means a lot to me.

my co-authors and colleagues, Magnus Garbrecht, Jens Jensen, Ivan Ivanov, Jun $L u$ for being always there for my scientific questions and for your help in the laboratories.

Pascal Ehret and Ileana Nedelcu, my collaborators in SKF. Thank you for your contributions and tries in the lab, for your phone meetings and discussions, trying to connect the dots between our fields, and of course, for giving faith in the project.

last here, but deep in my mind and heart, my family and Sophia. You show super human mental strength and support in these difficult years and situations. Be

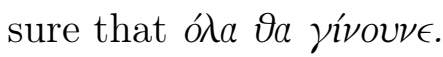




\section{Contents}

1 Introduction $\quad 9$

1.1 Challenges for roller bearings . . . . . . . . . . . . . . . 10

1.2 Motivation . . . . . . . . . . . . . . . . . . . 13

1.3 Carbon based thin films . . . . . . . . . . . . . . . . . . . 14

1.4 Carbon nitride $\left(C N_{x}\right)$ coatings grown with PVD . . . . . . . . . 17

2 Carbon nitride thin film synthesis by magnetron sputtering 21

2.1 Physics of sputtering and thin film deposition . . . . . . . . . . . . 21

2.2 Direct current, mid-frequency, and high power impulse magnetron

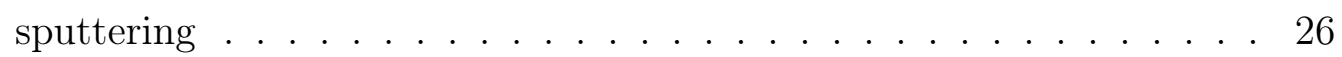

2.3 DC and pulsed negative bias voltage . . . . . . . . . . . . . . 29

3 Thin film characterization 33

3.1 Structural characterization . . . . . . . . . . . . . . . . . 33

3.1.1 Scanning Electron Microscopy $(\mathrm{SEM})$. . . . . . . . . . . . . 33

3.1.2 Transmission Electron Microscopy (TEM) . . . . . . . . . . . 34

3.1 .3 Focused Ion Beam (FIB) . . . . . . . . . . . . . . 38

3.1.4 X-ray Photoelectron Spectroscopy (XPS) . . . . . . . . . . 41

3.1.5 X-ray Reflectivity (XRR) . . . . . . . . . . . . 43

3.2 Mechanical characterization . . . . . . . . . . . . . . . 45

3.2.1 Profilometry . . . . . . . . . . . . . . . . 45

3.2.2 Nanoindentation . . . . . . . . . . . . . . . 47

3.3 Tribological characterization . . . . . . . . . . . . . . . . 50

3.3.1 Reciprocal friction and wear test . . . . . . . . . . . 50

4 Papers $\quad 55$

4.1 Paper I . . . . . . . . . . . . . . . . . . . . . 57

4.2 Paper II . . . . . . . . . . . . . . . . . . . . . . . 81 
5 Conclusions and upcoming work 101 5.1 Conclusions . . . . . . . . . . . . . . . . . . . . . . . 101

5.2 Upcoming work . . . . . . . . . . . . . . . . . . . . 104

6 Bibliography 107 


\section{Chapter 1}

\section{Introduction}

Thin films and rolling bearings are two different kinds of components that are both used in a vast variety of applications. Roller bearings are the heart of every component that rotates, from factory tools and machines to automotive industry and wind turbines. Their performance and reliability are of extreme importance for a number of reasons, such as cost and energy effectiveness, and safety. Rolling components are generally used under lubricated conditions and characterized by low to very low friction. With respect to the demanding conditions of roller operation, improvements are focused on their energy efficiency, failure prevention, and therefore expansion of their lifetime. In previous years, studies have been focused on the development of lubricants and additives, by exploring the lubrication mechanisms in the contact of the counterparts. However, enhancements on the overall operation of bearings can be achieved using thin films as coatings. The use of thin films for components can lead to higher efficiency and overall performance of the components, covering weaknesses of the lubricants. Thin films are used in a large range of applications, among them in semiconductor industry (electronics), green energy applications (i.e., photovoltaics, optical devices), printing technology etc. Thin films effectively change the characteristics of the substrates surface (for instance their resistance, reflectivity, surface energy, friction etc), by introducing a different compatible material on the component's surface, providing with improved or even new properties. The application of thin films on rolling or sliding components is directed by the needs and demands for using energetically more efficient materials with improved performance. This may potentially lead to a new expanded market, which will be exploiting the possibilities of two, nowadays different, fields: materials engineering and materials science, combining the knowledge from both. 


\subsection{Challenges for roller bearings}

Roller bearings consist of four different steel parts: a) rollers, b) cage, c) inner ring, and d) outer ring (Fig. 1.1). Common materials for roller bearings are high carbon chromium steel grading AISI 52100, several high-speed steels (for use at high temperature applications), stainless steel grading AISI 440C (for use at corrosive environments) and carburized hardened steels, whereas ceramic and silicon nitride bearings are also options for high performance applications.

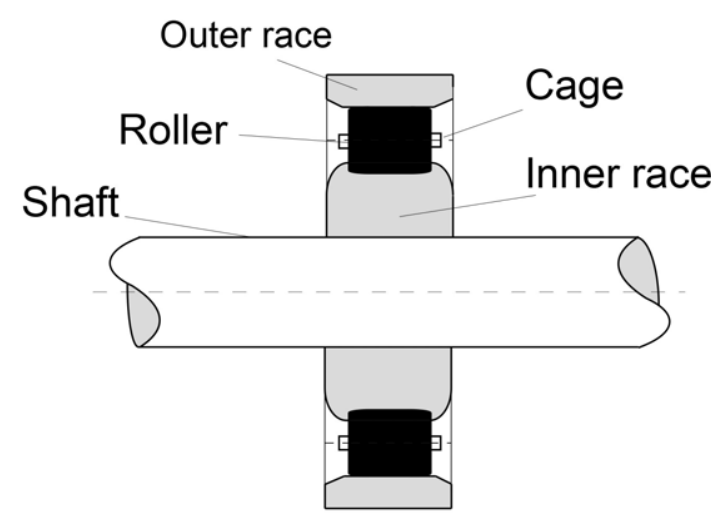

(a)

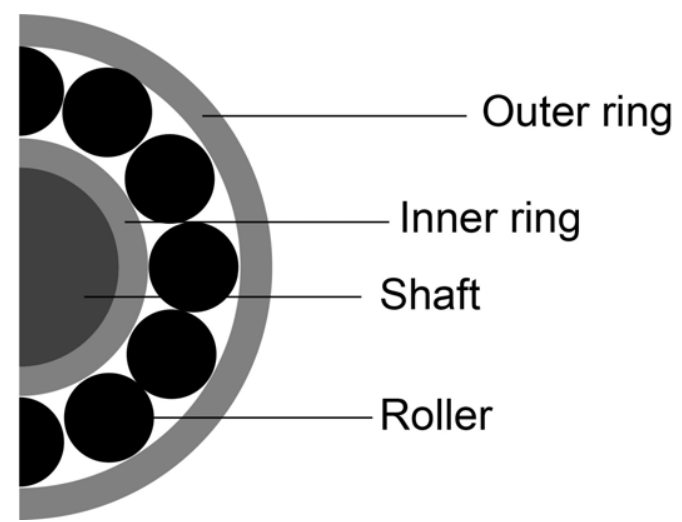

(b)

Figure 1.1: A cross-section of a roller bearing attached to a shaft (a) at $0^{\circ}$ and (b) $90^{\circ}$.

The contact between the rollers and the rings is characterized as "line" contact and it forms a more or less rectangular shape (Fig. 1.2), with contact pressures ranging between $0.5 G P a$ and $3 G P a$, depending on the application. The general operation of bearings is very well known and has been extensively described [1], [2]. An important complexity of the rolling mechanism is that roller bearings include a percentage of sliding during rolling, which can potentially cause failure such as seizure or corrosion. According to the elastohydrodynamic theory, a very thin lubricant layer is formed during the operation of a roller bearing, preventing adhesive and abrasive wear [1], [2].

A big problem for rolling bearings is fatigue wear, which causes pitting or spalling on the surface of the rollers. Pitting or micropitting (due to the size of the pits which are in $\mu m$ ranges) is regarded as a serious type of fatigue that reduces performance and lifetime of the component. Due to these failures, rollers need regular maintenance if not early replacement. The improvements in the performance and lifetime of the rollers results several benefits. For instance, the reduced service frequency 


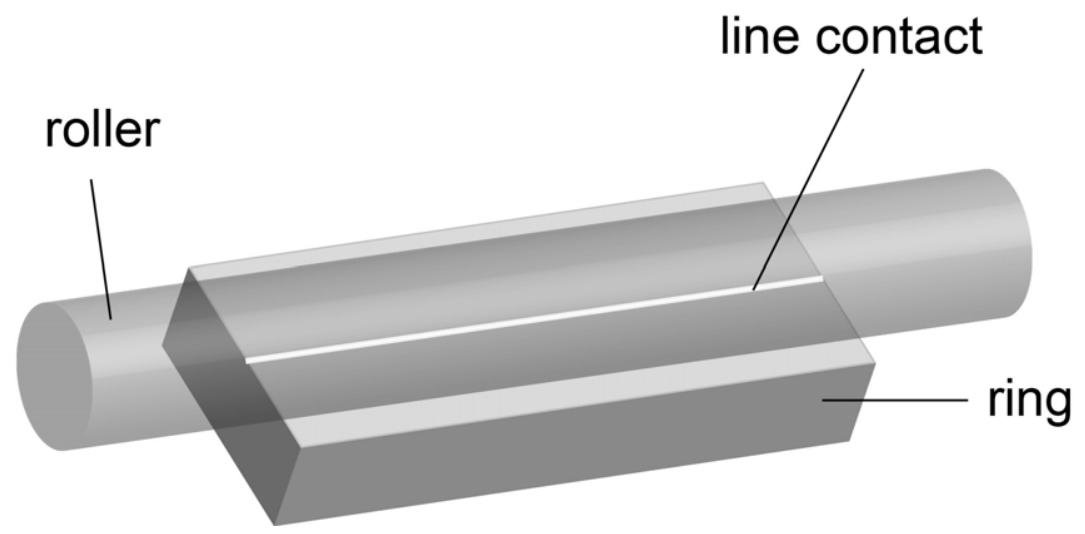

Figure 1.2: Schematic illustration of line contact between a roller and a part of a ring.

implies most cost-effective solution due to saving in materials (lubricants, additives, steel etc), directing the rolling mechanism to a "greener" operation. Moreover, an improved bearing performance results in a better overall performance of the component which makes use of the bearing.

The bearing lifetime can be extended by the application of a thin film coating on the roller or the rings surfaces. Thin films can contribute to lower friction at the contact between the roller and the rings. There are different types of thin films that are or can be used as protective and low friction coatings on bearings. Examples of materials used as coatings are $\mathrm{Au}, \mathrm{Pb}, \mathrm{Cu}, \mathrm{Cr}, \mathrm{MoS}_{2}, \mathrm{TiN}, \mathrm{TiC}$, and diamond-like carbon $(D L C)$. Some of them are soft and regarded as solid lubricants, i.e. $M o S_{2}$, due to their capability to operate without lubricant and they are successfully used in space applications [3]. Hard compound coatings, such as TiC, TiN or DLC are used in applications for low friction and wear resistance requirements, although they are grown by chemical vapour deposition techniques $(C V D)$ at elevated temperatures $\left(T>1000^{\circ} C\right)$. Using physical vapour deposition (PVD), TiN coatings have been also produced at temperatures as high as $600^{\circ} \mathrm{C}$ [4]. However, high temperatures are intolerable for bearing steels (due to a softening mechanism of steel, in which $\mathrm{C}$ is assumed to be interdiffused in the steel matrix), therefore $C V D$ techniques are unsuitable for coating depositions on roller bearings, turning the interest to $P V D$. $P V D$ usually employs significantly lower growth temperatures, ranging from room temperature $(R T)$ to $T<900^{\circ} \mathrm{C}$.

Another very important characteristic of rollers is surface roughness, where roller bearings with smoother surfaces exhibit longer endurance [1]. Surface roughness 
is a scale dependent parameter [1], [5] and defines the real contact between two counterparts. The contact area between two rough surfaces is less than the area between two smoother surfaces. This determines the degree of asperity interlocking and the debris creation in a wear test. Surface roughness has also different effects in rolling and sliding components. In sliding, asperity interlocking is more severe and phenomena, such as ploughing, asperity deformation and asperity adhesion affect friction and wear. In rolling, other phenomena dominate friction and wear behaviour, such as micro-slip effects within the contact, elastic hysteresis of the contacting materials, plastic deformation and adhesion effects in the contact. In a bearing rotation, where there is a small percentage of sliding, both rolling and sliding render the operation of the bearing more severe and complex. Roughness is also detrimental for the lubrication conditions in the contact, where in many cases specific roughness is desirable in order for the lubricant to be functional. The deposition of a coating with significantly lower roughness than the roughness of a roller or ring changes the contact conditions between the counterparts. Thus, it is challenging to understand the new contact mechanics and operation principles of the coated bearings under lubricated conditions.

The ultimate goal of coatings production suitable for bearings though is the reduction of friction and the enhancement of bearing wear resistance. Thus, the coating must serve as a low friction and wear resistance part of the bearing. To that direction, a suitable material for this purpose is DLC and its compounds. The use of carbon, as the base material for the films, has several advantages, such as the abundance and high availability of carbon, its low cost and many abilities for customization of such films. Carbon based thin films have been proved very attractive due to their mechanical and tribological properties and robust due to the variety of their structural characteristics [6] [7], [8] [9], [10], [11]. They exhibit low to very low friction coefficients and good wear resistance [10], [12], [13] high hardness (tetrahedral amorphous carbon, $t a-C$ films) and for some compounds high elasticity (for instance, fullerene-like carbon nitride, $F L-C N_{x}$ films) [12], and they are already used in application such as hard disk drives [11], [6], [14] and biomedicine [15]. One big problem of coatings, and especially carbon based, is their poor adhesion on steel substrates or steel components and this is reflected by the number of studies attempting to improve the adhesion between films and steel substrates [16], [17], [18], [19]. In applications where the applied forces and the film thickness are not prohibitive for the adhesion between the coating and the substrate, the problem can 
be solved with the deposition of an adhesion interlayer between the coating and the substrate. When the contact pressures are high and the film thickness increases, like in the case of roller bearings, the adhesion becomes a serious issue. Moreover, the cylindrical shape of rollers is also likely to influence the uniformity of the film, adding another implication to the already problematic nature of adhesion on steel.

\subsection{Motivation}

The motivation for this work stems from the challenges, regarding the depositions on roller bearings, and the fabrication of the coating materials. These challenges can be concluded as below:

- The substrate temperatures during the thin film growth must be less than $150^{\circ} \mathrm{C}$, due to the sensitivity of the bearing steel to temperatures higher than $170^{\circ} C$. At low temperatures, the $a-C N_{x}$ compound structure prevails over the structure of the very elastic and hard $F L-C N_{x}$ allotrope [20] [21]. Thus, $a-C N_{x}$ thin films, with the properties of $F L-C N_{x}$ thin films should be synthesized.

- The cylindrical shape of the rollers and the need for a uniform coating around the surface of the roller demands a special configuration of the deposition geometry.

- The poor adhesion between the steel substrates and the $C N_{x}$ films generaly, which is also influenced by special shapes, such as the cylindrical, when rollers are used as substrates.

- The further reduction of friction and wear of the bearing counterparts, and the prevention of premature failure met in uncoated bearings.

The aim of this work is to develop low friction and wear resistant carbon based coatings suitable for roller bearing applications. I try to solve the aforementioned problems in a two step plan. The first step is included in the present licentiate thesis and is the development of the coatings with the appropriate properties. The second step will be the coating deposition on rollers and the evaluation of their tribological performance. To overcome the problem of high temperatures, I will use PVD magnetron sputtering techniques, at temperatures $T<150^{\circ} \mathrm{C}$. The uniformity of the films deposited on rollers is expected to be achieved using rotation of the substrates. Thus, we will bind the aforementioned solutions and techniques to develop 
low friction and wear resistant $C N_{x}$ thin films, which were proven to be very resilient materials, due to their high hardness and high elastic recovery [12], [22].

In order to alter the structure and properties of the $C N_{x}$ films in all deposition techniques, confined by the demand for low temperature deposition, the substrate bias is varied, while other parameters were kept constant between the techniques. Furthermore, I used different deposition methods, which provide higher ionization (HiPIMS and MFMS) conditions, in order to explore their effects on the structure and properties of the films. The uniformity of the coatings around cylindrical shapes demanded depositions using a 3-fold rotation of the substrate table, with the engagement of special holders.

\subsection{Carbon based thin films}

Carbon (from Latin: carbo, "coal") is a remarkable element. It is symbolized with the capital letter $C$ and has atomic number 6 . It is nonmetallic, offering four electrons for the formation of covalent bonds with electronic configuration $[\mathrm{He}] 2 s^{2} 2 p^{2}$. In nature, carbon appears in the forms of diamond (clear, colourless) and graphite (black). Diamond (from the ancient Hellenic: áóa $\mu a s$, "unbreakable") is a natural form of carbon most commonly met in gemstones, having exclusively two interpenetrating face-centered cubic Bravais lattices. Diamond exhibits the highest hardness (bulk modulus of $433 \mathrm{GPa}$ ) of all known natural and synthesized materials up to date with a density of $3.52 \mathrm{~g} / \mathrm{cm}^{3}$ and remarkably high thermal conductivity (20

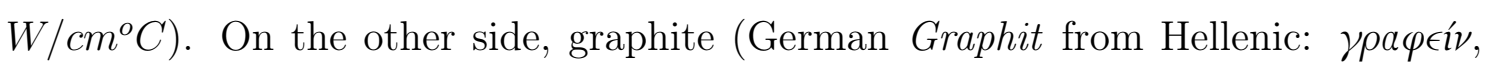
"to write" due to its use in pencils) is the most common form of natural carbon and its structure differs from that of diamond. The structure of graphite is not closepacked, but hexagonal and it exhibits carbon rings which lie in a flat plane, with other planes distributed in an $\mathrm{AB}$ AB AB... sequence above and below that plane. Nevertheless, graphite ore can also be found in nature as amorphous. Graphite is much softer in terms of hardness from diamond, although its loose interplanar bonding between the graphene sheets provides the material with very good lubricating properties. Carbon allotropes have been technically synthesized, with amorphous or tetrahedral amorphous forms, constituting diamond-like carbon $(D L C)$ materials. Carbon exists in the $\sim 90 \%$ of all known chemical substances and has the largest number of allotropes compared to any other element. Solid carbon materials exhibit also allotropic properties, depending on their microstructure. Apart from the solid 
bulk materials, $C$ is also used as building block for carbon based allotropes, including nanotubes, fullerenes, glassy carbons, carbon nanofibers, and numerous other compounds.

The bonds between $C$ atoms in a carbon-based compound include molecular orbitals, which are the combination of the atomic orbitals of $C$ atoms. When it is energetically possible, two $s$ atomic orbitals approach each other and overlap forming a new molecular orbital, namely $\sigma$-orbital. Respectively, the combination of two $p$ atomic orbitals gives a $\pi$ molecular orbital. If an $s$ and a $p$ atomic orbital are combined, then three different molecular hybridized orbitals are formed, namely, $s p^{3}$, $s p^{2}$ and $s p$ hybrids. The concept of hybridization was proposed by Linus Pauling in 1931 and $C-C$ bonds include hybridized orbitals to form either natural or synthetic types of $C$ (i.e., diamond, graphite, $D L C$ etc.).

The four valence electrons of $C$ can form three hybridization states, the $s p^{3}$ (with four hybridized $\sigma$ orbitals), $s p^{2}$ (with three hybridized $\sigma$ orbitals and two $\pi$ orbitals) and $s p$ (with two $\sigma$ orbitals and four $\pi$ orbitals). $s p^{3}$ hybridized orbitals with tetrahedral coordination are met in diamond, $s p^{2}$ hybridized orbitals are mainly met in graphitic configurations, while $s p$ orbitals form configurations similar with alkalines (Fig. 1.3). In an $s p^{3}$ hybridized carbon, the four valence electrons form $\sigma$ orbitals, which point towards the corners of a tetrahedron and a network of $s p^{3}$ bonds has a 3 dimensional configuration (Fig 1.3(a)).

a $\mathrm{sp}^{3}$

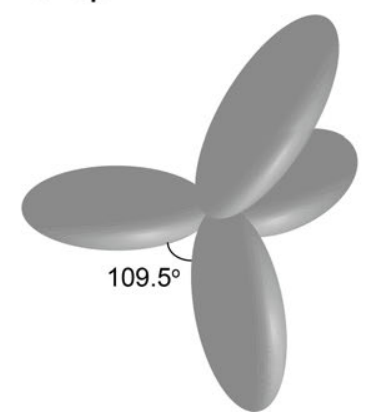

b $\mathrm{sp}^{2}$

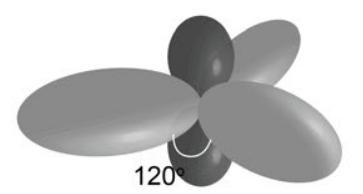

c sp

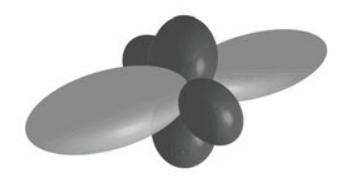

Figure 1.3: (a) $s p^{3}$ hybridization state of $\mathrm{C}$ atoms, like in diamond structure, (b) $s p^{2}$ hybridization state of $C$ atoms, like in graphite, and (c) $s p$-hybridization state of $C$ atoms, like in alkynes.

The high bulk modulus of diamond is a direct consequence of the $s p^{3}$-bonding. The $C$ - $C$ bonds exhibit a bond energy of $3.9 \mathrm{eV}$ with a bond length of $154 \mathrm{pm}$. 
Carbons with an $s p^{2}$-rich network, have three electrons participating in three planar $\sigma$ bonds with an angle of $120^{\circ}$, while the fourth electron has a $\pi$ orbital perpendicular to the $\sigma$ orbitals (Fig 1.3(b)). These hybridization states are met in graphite and graphene, with enhanced in-plane strength, due to a shorter $C-C$ bond of $133 \mathrm{pm}$ and strength of $\sim 7.5 \mathrm{eV}$. In $s p$-hybridized bonding states carbon forms two orthogonal $\pi$ orbitals and two linear $\sigma$ orbitals, comprising a bond length of $120 \mathrm{pm}$ and strength of $9.9 \mathrm{eV}$ (Fig 1.3(c)).

The production of $D L C$ films was done in early 1950's by Schmellenmeir [23], although $D L C$ coatings attracted attention almost two decades later, with the work of Aisenberg and Chabot [24]. DLC films comprise a large family of amorphous carbon-based materials with a variety of allotropes. They consist of a mixture of $s p^{2}$ and $s p^{3}$ carbon structures, usually with $s p^{2}$ bonds embedded in an $s p^{3}$ matrix.

A phase diagram proposed by Robertson summarizes the expressed microstructures of $D L C$ materials (Fig. 1.4) [7]. $D L C$ are mainly amorphous materials divided to $\mathrm{H}$ containing and $\mathrm{H}$-free carbons. Both $D L C$ films can be met in different amorphous types; tetrahedral amorphous $(\mathrm{ta}-\mathrm{C})$ and hydrogenated tetrahedral amorphous carbon $(t a-C: H)$, amorphous carbon $(a-C)$ and hydrogenated amorphous carbon $(a-C: H)$, sputtered amorphous carbon and hydrogenated sputtered amorphous carbon as well as glassy or graphitic carbon.

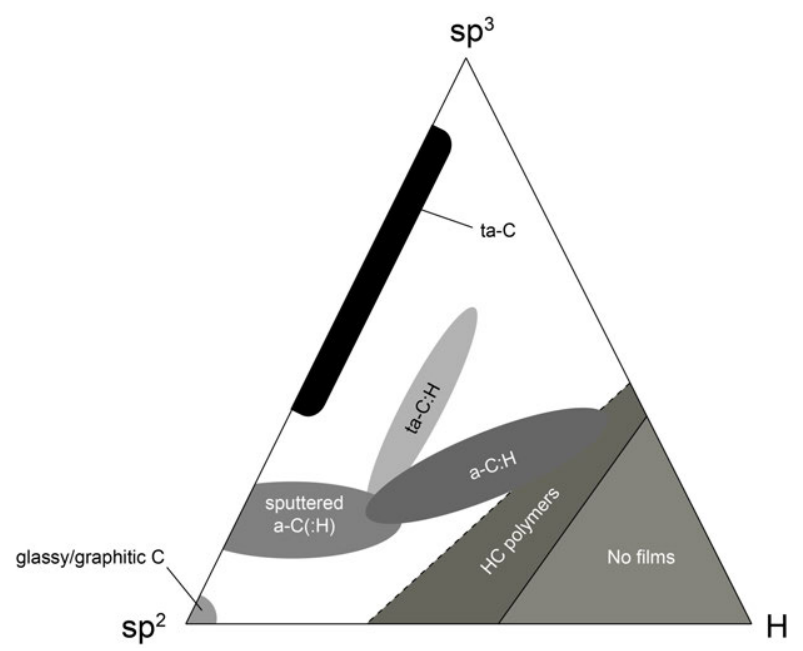

Figure 1.4: Ternary phase diagram of $a-C$ and $a-C(: H)$ materials. Figure adopted from [7].

This subdivision depends on the $\mathrm{H}$ content and the amount of $s p^{3}$ and $s p^{2}$ hybridization states in $D L C$ film [25], [7]. As the amount of $s p^{3}$ hybridized states increases, $t a-C$ structure prevails, with a corresponding influence on the mechanical 
properties of the films, where very hard materials are formed. If the $s p^{2}$ bonding dominates the $D L C$ films exhibit more graphitic structures (due to the configuration of $s p^{2}$-hybridization, as described above), forming $a-C$ and $a-C: H$ films. Another category of $D L C$ materials is carbon nitride $\left(C N_{x}\right)$ films, which contain a high $s p^{2}$ fraction, mainly induced by $N$ incorporation in the $C$ matrix. The introduction of $N$ in a carbon matrix promotes cross-linking and bending of basal planes of the matrix, providing enhanced elasticity to the films [26]. Finally, an emerging category is also the metal/amorphous $(a-C: M)$ composite films, where a metal is embedded in the $a-C$ matrix.

\subsection{Carbon nitride $\left(C N_{x}\right)$ coatings grown with PVD}

The introduction of a small amount of foreign element in the $C$ matrix is called doping of $D L C$. Doping can be achieved using several elements, such as $N, F, P$, $S, S i$ or metals like $\mathrm{Ti}, \mathrm{Cr}, \mathrm{Ta}, \mathrm{W}$ etc. and can alter the structure and properties of the initial film. Nitrogen is an element with similar hybridization states as $C$. It can also form covalent bonds, having an electronic configuration $[H e] 2 s^{2} 2 p^{3}$. It also forms $s p^{3}$ and $s p^{2}$-hybridized states, but the extra electron introduces some changes regarding the bond angles in $s p^{3}$ configuration and also results in two configurations when it bonds with $s p^{2}$ states (Fig. 1.5). The $s p^{3}$ formation is similar to that of ammonia, leaving an unbonded pair of electrons, while the $s p^{2}$ states are similar to that of (i) pyridine or (ii) to a 3 -fold coordination substituting a $C$ atom in a graphitic site [20].

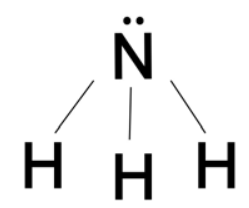

$\mathrm{sp}^{3}$

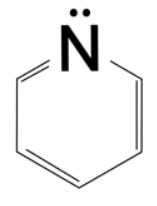

$\mathrm{sp}^{2}$

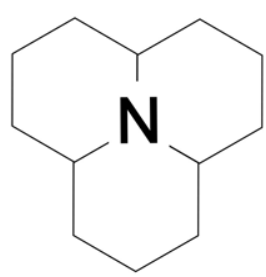

$\mathrm{sp}$

Figure 1.5: (a) $s p^{3}$ hybridized bonds of $N$ atoms, like in ammonia, (b) $s p^{2}$ hybridized bonds of $N$ atoms, like in pyridine and (c) $s p$ hybridized bonds of $N$ atoms with 3-fold coordination with $C$ atoms.

Carbon nitride is a carbon allotrope material, which attracted a lot of attention when Liu and Cohen [27], [28] predicted the metastable $\beta$ phase of $C_{3} N_{4}$ in 1990, 
which in theory exhibited a bulk modulus (427 $\mathrm{GPa}$ ) very close to that of diamond (433 GPa [29]). The synthesis of $\beta-C_{3} N_{4}$ was not successful up to date, although other interesting materials were synthesized, such as elastic and resilient $s p^{2}$-rich amorphous carbon nitride $a-C N_{x}$ and fullerene-like carbon nitride $F L-C N_{x}$ compounds [30], [31], [32], [33], [34], [10]. The synthesis of amorphous carbon nitrides are dated earlier than fullerene-like carbon compounds, because all tries for the deposition of $\beta-C_{3} N_{4}$ ended in the growth of amorphous phases. In 1995, the first fullerene-like $C N_{x}$ compound was synthesized by Sjöström et al. [35] in Linköping University, a material that was further investigated experimentally by Hellgren et al. [36], [37], Neidhardt et al. [34], [22] and Schmidt et al. [21], [38]. Simultaneously, theoretical studies performed by Gueorguiev et al. [26], [39], showed the possible structural evolution of $C N_{x}$ compounds, described by cross-linkage of individual graphene sheets and bending basal planes extended in a 3-dimensional network.

Both carbon allotropes were synthesized by several PVD deposition techniques, such as radio frequency magnetron sputtering (RFMS) [40], [41], direct current magnetron sputtering (DCMS) [42], [36], [43], [21], high power impulse magnetron sputtering (HiPIMS) [21], [38], pulsed laser deposition (PLD) [44], [10], filtered pulsed cathodic arc deposition [10], [45] and ion beam assisted deposition (IBAD) [46]. Magnetron sputtered $C N_{x}$ films show clear dependency on the deposition temperature. For $C N_{x}$ films deposited with DCMS at low substrate temperatures $\left(T<200^{\circ} C\right)$, amorphous and dense films were grown, while increasing the substrate temperature, FL microstructure prevailed. The $N$ content in the films and the degree of $\mathrm{N}$ incorporation also depend on substrate temperature. For films grown at $T<200^{\circ} \mathrm{C}, \mathrm{N}_{2}$ fraction did not influence the film growth, while at $T>200^{\circ} \mathrm{C}$, chemical sputtering interactions took place at the substrate's site [37], [20]. For $C N_{x}$ films deposited by HiPIMS, similar temperature dependency was found, where at elevated substrate temperatures the $F L$ structure becomes dominant [21]. However, chemical sputtering interactions were not so intense and did not influence the film formation to a high degree [21]. $a-C N_{x}$ thin films have been deposited by several research groups with the use of different magnetron sputtering techniques, mainly RFMS and DCMS. Kleinsorge et al. [47], Hellgren et al. [36] and Ferrari et al. [31] have thoroughly studied the bonding of $N$ within $C$ matrix in $a-C N_{x}$, concluding that $N$ incorporation promotes $s p^{2}$ hybridization states. This has immense effects on the mechanical response of the films, especially their elasticity. It is believed that the cross-linkage and bending of the basal planes are responsible for the increased 
resiliency of the $F L-C N_{x}$ films. $a-C N_{x}$ films exhibit also enhanced resiliency and elasticity, but not to the same degree as the $F L-C N_{x}$ compound, which can potentially reach $100 \%$ of elastic recovery after indentation [22].

The attractive mechanical properties as well as the low friction exhibited by both $a-C N_{x}$ and $F L-C N_{x}$ thin films, render them as candidates for sliding and rolling components like roller bearings. Nevertheless, the high growth temperatures needed for the synthesis of $F L-C N_{x}$ films, turn the attention to the further exploration of the $a-C N_{x}$ films applicable for bearings. 


\section{Chapter 2}

\section{Carbon nitride thin film synthesis by magnetron sputtering}

\subsection{Physics of sputtering and thin film deposition}

The processes for growth of thin films must be conducted in high or ultra high vacuum conditions to avoid contamination of the films and increase ionization of the working gas. A noble gas $(A r, N e, X e$ etc.) -the working gas- is let into the vacuum chamber as the sputtering agent, because the atoms are non reactive and do not contaminate the surface of the target. A potential is applied between the cathodes and the grounded chamber walls, which act as anode, in order to accelerate free electrons and excite the gas molecules. This potential difference accelerates a small amount of background electrons and ions, which is present in the chamber, in the cathode field. The electrons are repelled from the cathode, causing ionization of the working gas if they have sufficient energy and a glow discharge is initiated. The positively charged gas ions (i.e., $A r$ for convenience) are then attracted towards the cathode and collide on the target. Due to their momentum, Ar ions sputter neutral atoms from the target, although a number of scattered particles are also removed from the target, namely secondary electrons, reflected ions and neutrals, and photons, creating a plasma; an ionized phase of the sputtered gas, with glow colors characteristic of each sputtered gas. The secondary electrons ionize more working gas atoms, which impinge on the target and produce more secondary electrons, contributing to an avalanche process. The plasma is sustained when enough secondary electrons are generated and contribute to the ionization processes of the working gas, accelerated away from the target, thus at very low 
or very high pressures, plasmas are not possible to be sustained. According to Paschen's law, between a few hundreds to a thousand volts, the discharge can be self-sustained [48]. The neutral target atoms travel from the target's surface and are deposited on the substrate, forming a film consisting of the target material.

Thin film growth by magnetron sputtering techniques is overwhelmed by sputtering phenomena, where the term "sputtering" can describe several ion-surface interactions. Sputtering is the process in which atoms are ejected from the surface of a solid material (target), due to collisions between projectile/recoil atoms and atoms of the surface of the target. Impinging ions create a collision cascade at the area of incidence and when they have sufficient energy, surface atoms are removed from the target (Fig. 2.1). Surfaces in plasmas are exposed in impinging ions and are subjected to several different ion-surface interactions, such as ion and recoil implantation, atom displacement, backscattering, generation of secondary electrons, atom redeposition, photon emission, generation of collision cascades, and/or ion/neutral reflections (Fig. 2.1).

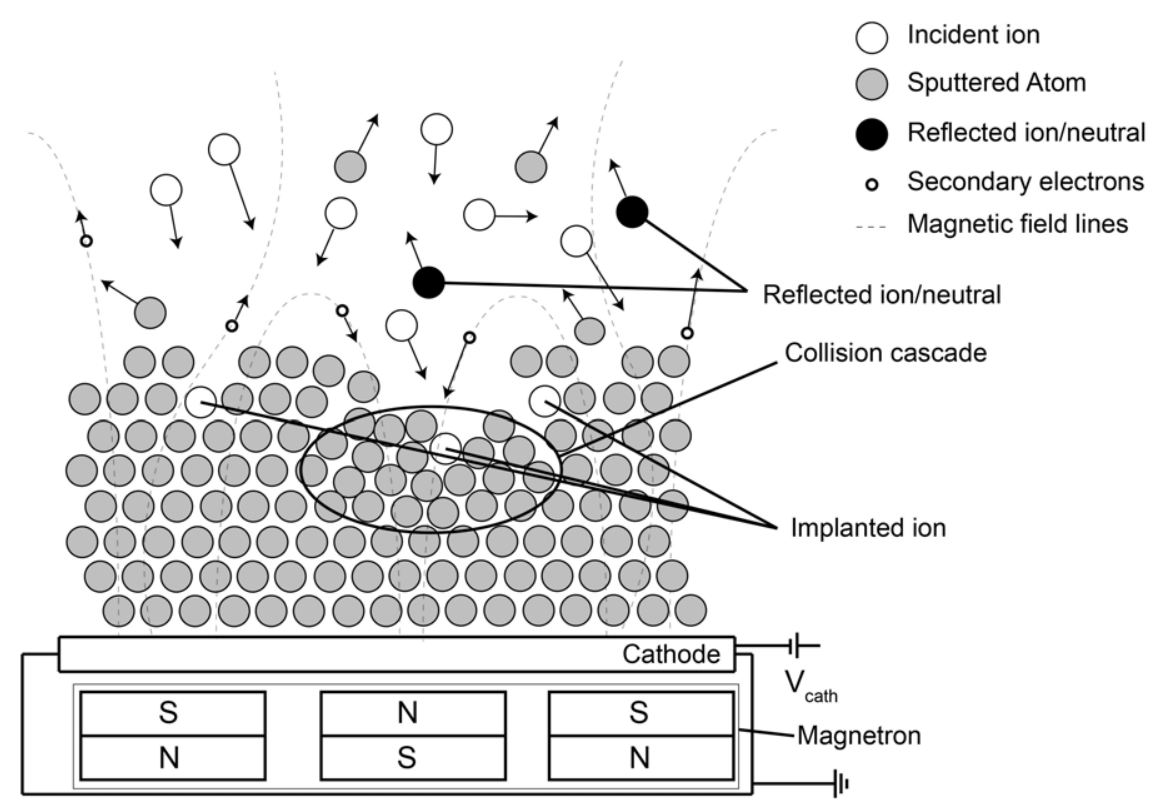

Figure 2.1: Schematic illustration of sputtering interactions on a theoretically pure target containing only one material element. Cathode and the configuration of an unbalanced magnetron are shown below the target. The dimensions of the target and ions/atoms are exaggerated and do not correspond to the cathode and magnetron sizes.

The ion breaks up the sequence of atoms of the solid surface causing sputtering 
and scattering of some atoms backward. The sputter yield of a target can thus be determined as:

$$
S=\frac{\text { Number of sputtered atoms }}{\text { Incident ion }}
$$

and is a measure of the efficiency of the sputtering process or the average amount of particles removed from the target per incoming ion. The sputter yield depends on the type and energy of the impinging particle, the incident angle as well as the target material.

Very important components incorporated in sputtering techniques are magnets, in order to improve the ionization conditions. Magnets are placed behind the cathode plates, with different configurations and a magnetic field is applied. The magnetic field lines confine the motion of secondary electrons in the region of the cathode and according to the magnetic field lines. The time that secondary electrons spend in the vicinity of the target is increased and the plasma is easier maintained. The collision probability between the confined electrons and the plasma species becomes higher, and thus higher plasma densities can be achieved. There are three different magnetron configurations, namely the balanced, the unbalanced type I, and unbalanced type II magnetron. In a balanced magnetron, all magnets have the same strength, while in an unbalanced magnetron configuration, the magnets have different strength. Type I and type II refer to which magnets are stronger. The unbalanced magnetron configuration is a very effective way to enhance the ion bombardment. In an unbalanced magnetron, a selective strengthening of the magnetic field at the target ends can be chosen, so that more of the secondary electrons escape from the confinement, increasing the plasma ionization. This configuration increases also the current at the substrates compared to a balanced magnetron, moreover lower applied voltages can be used in order to sustain the plasma and achieve the desirable ionization conditions.

When a reactive gas (for instance $\mathrm{N}_{2}, \mathrm{O}_{2}$, methane etc.) is involved as working gas in the sputtering process, then the process is called reactive magnetron sputtering. The reactive gas can be mixed with the inert working gas or can dominate the sputtering process. Compound materials can be formed such as oxides $\left(\mathrm{Al}_{2} \mathrm{O}_{3}, \mathrm{SiO}_{2}\right.$ etc.), nitrides ( $T i N, A l N, S i_{3} N_{2}, C N_{x}$ etc.), carbides (TiC, SiC etc.), sulphides, oxycarbides and oxynitrides. $C N_{x}$ films in $P V D$ are deposited in a mixture of $N_{2} / A r$ gas or entirely in $N_{2}$ atmosphere. When $N_{2}$ flows in the chamber during the sputtering process, $N^{+}$and $N^{2+}$ ions can be formed. These ions are involved in the 


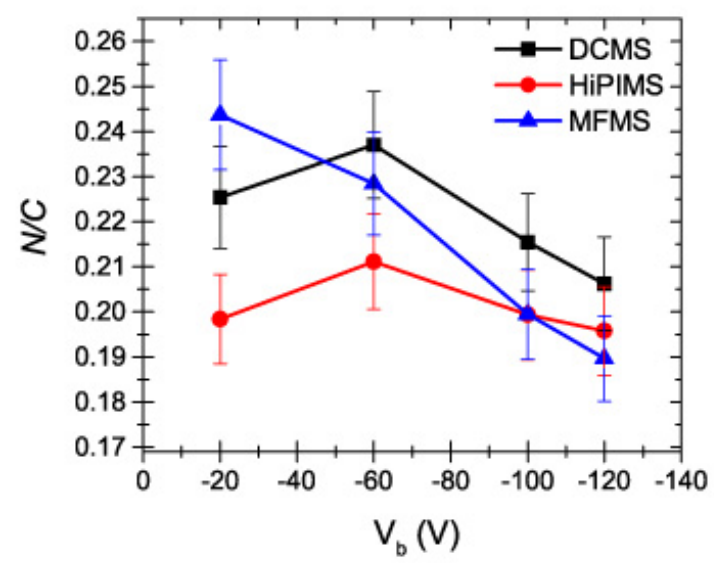

(a)

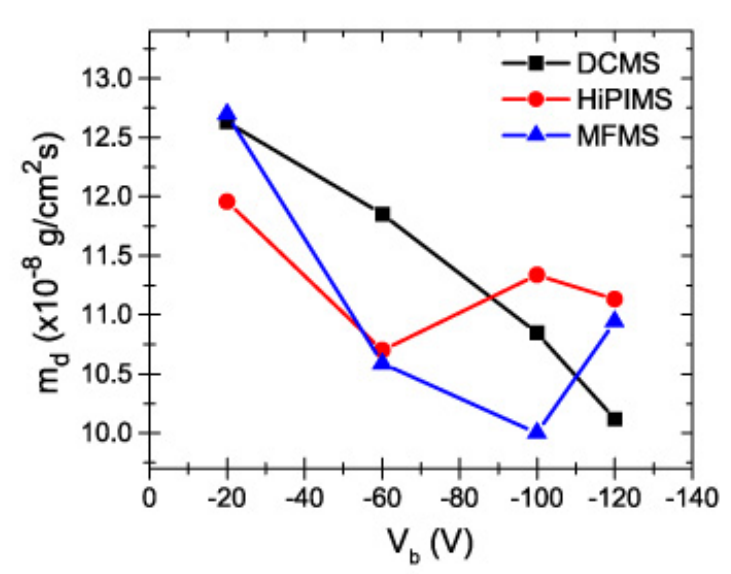

(b)

Figure 2.2: (a) $N / C$ ratio and (b) deposited mass per area and time of $C N_{x}$ films deposited by MFMS (triangles), HiPIMS (circles), and DCMS (squares) as a function of $V_{b}$.

target sputtering sequence, causing dangling bonds at the target surface, followed by passivation with atomic $N$ species or $C_{x} N_{y}(x, y \leq 2)$, if no recombination with $C$ or $H$ occurs. Volatile $C N$-species can form and desorb from the target surface and travel through the plasma to the substrates [37]. This is a process referred to as chemical sputtering of the target.

A chemical sputtering process is also observed at the substrates during the reactive sputtering of graphite targets with $N_{2}$. This interactions have been previously observed by Hellgren et al. [37], Hammer et al. [49], Kaltofen et al. [50], when DCMS is used for the synthesis of $C N_{x}$ films and by Schmidt et al., [38] when HiPIMS is used as growth process. For films grown by DCMS in the past, the chemical sputtering on the film was pronounced at elevated flow ratios $\left(N_{2} / A r>0.5\right)$ and for temperature $T>200^{\circ} \mathrm{C}$, where the $N$ content in the films did not exceed $25 \mathrm{at} \%$. This accounts for removal of $N$ from the growing film, which is confirmed by lower growth rates and $N$ incorporation in the films. Chemical sputtering was found to be suppressed when HiPIMS was used.

Regarding the $C N_{x}$ films investigated here, chemical sputtering is assumed to be present. Nevertheless, re-sputtering of $N$-containing species was mainly observed (Fig. 2.2(a)) due to high energy ions at higher $V_{b}$, as $N$ was found reduced in films deposited by MFMS and DCMS at higher $V_{b}$. Re-sputtering was found to be suppressed in HiPIMS, since these processes presented constant $N / C$ ratio with 
increasing $V_{b}$. The deposited mass per area and time, $m_{d}$ (in $\left.\mathrm{g} / \mathrm{cm}^{2} s\right)$, is shown in Fig. 2.2(b) for $a-C N_{x}$ films deposited by MFMS, HiPIMS, and DCMS. In the case of MFMS, $m_{d}$ of the films presents a minimum at $V_{b}=100 \mathrm{~V}$, while $\rho_{c}$ of these films increases linearly with increasing $V_{b}$ from $\sim 1.84 \mathrm{~g} / \mathrm{cm}^{3}$ to $\sim 2.2 \mathrm{~g} / \mathrm{cm}^{3}$. This shows that re-sputtering interactions are present up to $V_{b}=100 \mathrm{~V}$, although the increase of both $m_{d}$ and $\rho_{c}$ at $V_{b}=120 \mathrm{~V}$ implies a suppression of re-sputtering. When HiPIMS is used, $m_{d}$ does not present a certain trend, indicating rather a balance between resputtered desorbed species, and absorbed $C, N$, and $C N$ species, which contribute to film formation (see also Paper I). For MFMS and DCMS modes, re-sputtering follows different trends, showing that these techniques may have their limitations regarding the applied bias voltages. With both of them suffering re-sputtering with increasing $V_{b}$, the deposition rates will steadily be decreasing, as $V_{b}$ increases. Therefore, at some point, the deposition rate will be less than the re-sputtering rate and the film formation would be impossible, leading to severe sputtering of the substrates from high energy ions. In HiPIMS mode, re-sputtering is not that pronounced, however the operation of the cathode-target assembly in this technique can usually lead to severe arcing, since it is governed by very high currents through the cathode-target. One should take care that during reactive-HiPIMS of graphite targets avoids the combination of parameters that cause arcs, in order to preserve the target and to prevent defects in the films, such as macroparticles. Arcing of graphite targets in reactive magnetron sputtering processes may be a consequence of the high ionization potential of $C$ and possible contamination layers on the target surface which affect the sputtering process. Macroparticles, consisting mainly of $C$, escape during arcing of the target in any time during HiPIMS deposition and may land on the substrates. These macroparticles can be as large as the film thickness with several $\mu m$ diameter. An example of a macroparticle incorporating during the reactive-HiPIMS deposition of $C N_{x}$ is shown in Fig. 2.3(a). This macroparticle extends almost from the middle throughout the film, meaning that it was generated and was deposited on the film sometime during the middle of the process. Macroparticles may be generated during DCMS and MFMS processes as well, although the problem is not so pronounced, resulting to much smoother and defect-free surfaces. It is assumed that especially the configuration of MFMS mode allows for arc-free processes. Fig. 2.3(b) shows a defect in the film which does not consist of a macroparticle, but it could be generated by the deposition of a macroparticle of smaller size during the initial deposition process. In this case, the macroparticle was deposited on the film and 
the deposition at that point continued with different route, resulting this growth in the film. Several such defects are observed on the surface of this film.

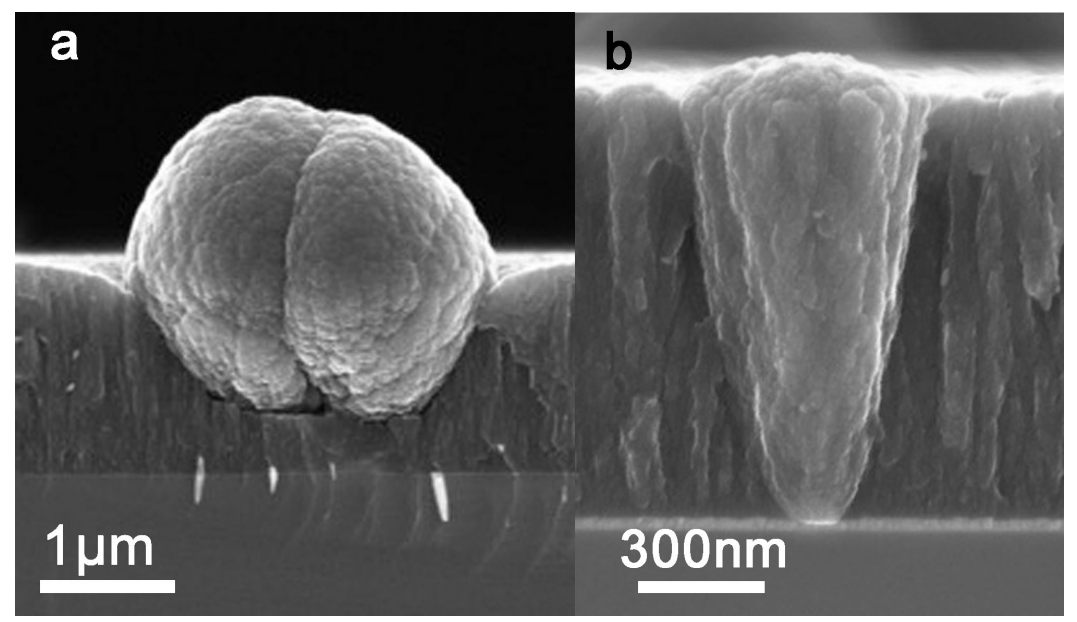

Figure 2.3: (a) Cross-sectional SEM image of vizualising a macroparticle that was incorporated in the film during deposition and (b) nodular growth of a defect grown after a smaller macroparticle was initially deposited on the substrate.

\subsection{Direct current, mid-frequency, and high power impulse magnetron sputtering}

DC magnetron sputtering is a widely used technique both in research and industry due to its fairly acceptable deposition rates and is well understood process for $C$ based and specifically for $C N_{x}$ films. Depending on the deposition parameters (i.e., total gas pressure, gas composition, substrate temperature, average cathode power), the microstructure and the properties of the $C N_{x}$ films can be altered [22].

Mid-frequency magnetron sputtering (MFMS) provide of an evolution of the conventional DCMS, using pulsed power supplies (AC) for the generation of the plasma. Initially, MF was used to deposit insulating materials, which were subjected to unstable discharges with arcing. Arc generation causes defects in the films, such as macroparticles. In an effective MF configuration, two operating targets are necessary, where one target works as cathode and the other as anode, altering their polarity every half cycle. The operating frequency can vary, depending on the deposition system, from low radio frequencies $(\mathrm{RF})$ of $f \approx 50 \mathrm{~Hz}$ as high as $f \approx 1 \mathrm{MHz}$. High RF frequencies produce high quality films, however, the deposition rates have 
been proven very low for commercial applications. In MF conditions, frequencies usually are in the range of few hundreds of $k H z(50-250 \mathrm{kHz})$. The pulse shape of the MF processes is shown in Fig. 2.4, for the configuration of dual magnetron and bipolar pulsing, described above.

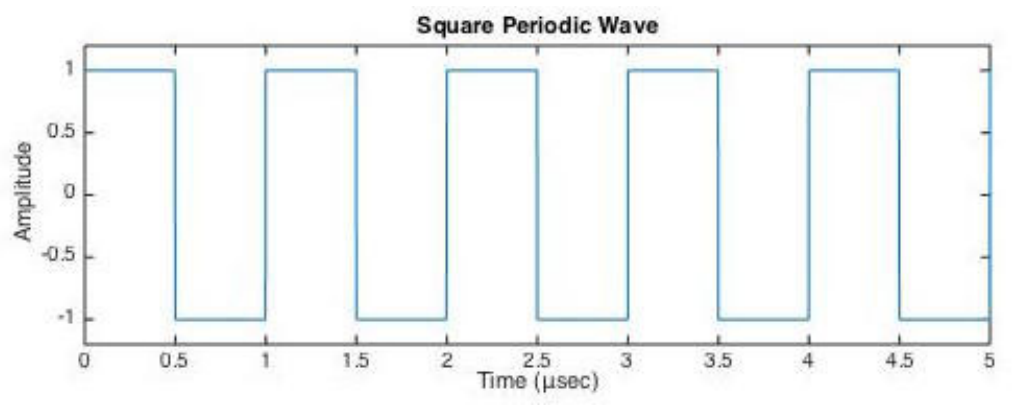

Figure 2.4: An example of a square pulse, like those used by power supplies in MF deposition processes. The axis labelling and the pulse lengths as well as pulse-on and pulse-off times are arbitrary.

MF produces tetragonal pulses with the pulse-on times of the cathode equal to the pulse-off times of the anode. Duty cycles are usually in the region of $50 \%-50 \%$ and are given from:

$$
D=\frac{t_{\text {pulse }}^{\text {ON }}}{t_{\text {pulse }}}=f \cdot t_{\text {pulse }}^{O N}
$$

where $t_{\text {pulse }}^{O N}$ is the pulse-on time, $t_{\text {pulse }}$ is the pulse length and $f$ is the operating frequency.

High power impulse magnetron sputtering (HiPIMS) is also a pulsed process and an evolution of DCMS, although the operation principle differs significantly from DCMS and MFMS. In HiPIMS the sputtered target operates, like in DCMS processes, as cathode and the chamber walls operate as anode. Pulsed high power supplies are used to generate high current pulses and deliver high amounts of energy to the plasma. These high peak currents are two orders of magnitude higher than the average target currents in a DCMS process, resulting to high plasma densities (in the order of $10^{18} \mathrm{~m}^{-3}$ [51], when for DCMS high respective values are in the order of $10^{16} \mathrm{~m}^{-3}$ ). The high peak currents and plasma densities increase the possibility for ionization and thus for sputtering of material from the target. Kouznetsov et al., 
[52] investigated $C u$ targets in HiPIMS conditions and found that the ion fluxes in HiPIMS were two orders of magnitude higher than in a DCMS process. The pulses in HiPIMS processes exhibit usually low duty cycles of $<10 \%$ and frequencies $<10$ $k H z$. It has been demonstrated that HiPIMS often leads to deposition of dense and homogeneous thin films, where the targets operated in either metallic or reactive mode, although a reduction of the deposition rate has been also observed compared with DCMS of the same material targets [53].

DeKoven et al. [54] had reported the deposition of $D L C$ films using HiPIMS back in 2003, while Hecimovic and Ehiasarian [55] demonstrated that the plasma characteristics and the sputtering of $C$ targets in HiPIMS mode differs from sputtering of other metal targets. $C N_{x}$ thin films have been deposited in $N_{2} / A r$ by reactive HiPIMS later in 2012 by Schmidt et al., where amorphous films prevail at low growth temperatures and fullerene-like structures were produced at high temperatures [21], following the general course mentioned above for DCMS. Lower deposition rates were also reported for $C N_{x}$ film synthesis with HiPIMS compared to DCMS [21]. An extensive and thorough comparison regarding the plasma characterization and the film growth, between DCMS and HiPIMS depositions of $C N_{x}$ can be found in [21] and [38]. The effects of reactive DCMS on $C N_{x}$ film synthesis are well understood. Nevertheless, the effects of HiPIMS and MFMS of graphite targets, when operating in metallic or reactive mode, on pure $C$ and $C N_{x}$ film synthesis, microstructure, and properties need to be further explored.

In this study, an industrial deposition chamber is used for the growth of $C N_{x}$ thin films, using either MFMS, HiPIMS or DCMS under similar growth conditions. The chamber configuration is illustrated in Fig. 2.5, where at the position of each cathode/target is indicated in which mode each cathode can operate. For our depositions in MFMS processes, cathodes indicated as $M F^{a}$ were working against each other, while during HiPIMS processes, one of the two HiPIMS cathodes was employed. In DC mode, cathodes $D C^{a}$ were used. The table carried separate holders, where each holder carried separate steel rods for the mounting of the substrates. Thus, a 3 -fold rotation with $1 \mathrm{rpm}$ of the substrates was used during the depositions, which guaranteed the uniformity of the coating, mimicking the deposition process on rollers. 


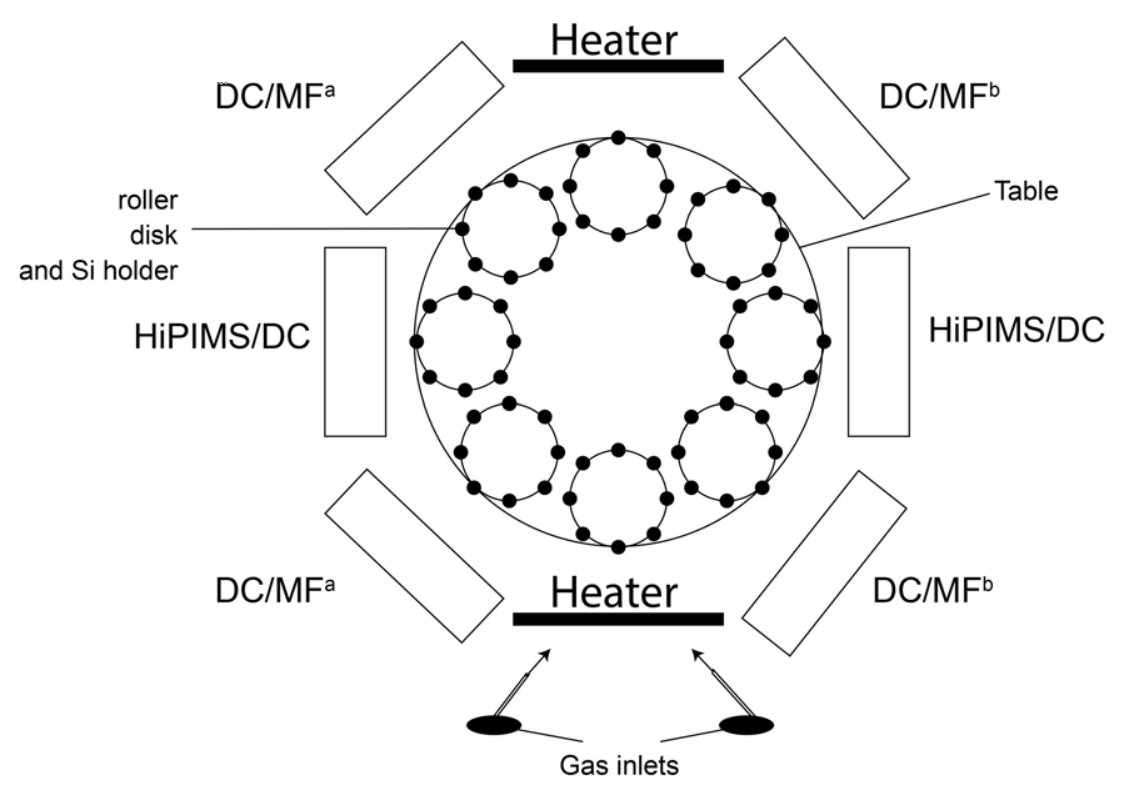

Figure 2.5: Illustration of the top view of the deposition chamber. The components of the chamber are also shown, such as cathodes, table and holders with 3-fold rotation, gas inlets, and heaters.

\subsection{DC and pulsed negative bias voltage}

Ion bombardment of the substrates during film growth is crucial. The application of a negative bias voltage (DC or pulsed) at the substrate is a well-known method to increase the flux/energy of ions incident at the grown film surface. For $C N_{x}$, Laskarakis et al. [56] investigated the structural changes that low and high energy ions induced in the $C$ matrix, where $N$ is incorporated with different manner, while Neidhardt et al. [22] showed that small variation of the ion energies from $25 \mathrm{eV}$ to $40 \mathrm{eV}$, altered the mechanical properties of high-temperature $F L-C N_{x}$ films, producing harder and more elastic materials. However, there is no study, at our best knowledge, showing which are the changes that different and higher than $40 \mathrm{eV}$ ion energies trigger to the morphology of low-temperature $C N_{x}$ films, either amorphous or fullerene-like. Petrov et al. have investigated the effects of different ion energy on the microstructure and morphology of reactively magnetron sputtered polycrystalline TiN films [57]. They have shown that low ion energies result to somewhat open structures, with intercolumnar and intracolumnar voids and attributed this behavior to limited surface diffusion. At higher ion energies $(>100$ $e V)$, more homogeneous films resulted, although increased defect density and impurity incorporation, such as inert gas species, appeared. It is crucial to investigate the 
morphological and microstructural changes occurring to $C N_{x}$ films for depositions at different ion energies, since morphology is detrimental for the mechanical and tribological properties of thin films. Moreover, the constraint of low-temperature depositions, confines the customization of the morphologies of the films through variations of temperature.

Density and homogeneity are pre-requisites for the formation of hard and wear resistant thin films. Thus, except from the bias voltage, the ionization conditions can be altered using different deposition mode, which operates with different type of bias voltage. Each different technique produces films with different morphologies (from under-dense and columnar to dense and homogeneous), depending on these ionization conditions and the special operation of the bias voltage, employing different duty cycle. Significant differences arise in the $C N_{x}$ film morphology when different type of substrate bias voltage is applied. Furthermore, different ionization condition, namely MF, HiPIMS, and DC bias voltage, influence differently the way that the ion energies are delivered to the substrates and the evolution of their final film structure. The bias duty cycles are 44\%, 100\%, and 6\% for MF, DC, and HiPIMS bias voltage, respectively. Hence, different amount of ions results on the substrates in each case and for different time intervals. HiPIMS processes allow for smaller fraction of ionized species to result on the substrate per time unit, due to the very low bias duty cycles compared to the other two technique. Fig 2.6 shows fracture cross-section scanning electron microscopy (SEM) images of $C N_{x}$ films deposited by MFMS, HiPIMS, and DCMS as a function of $V_{b}$.

Interestingly, at $V_{b}<20 \mathrm{~V}$ columnar structures with intercolumnar voids are observed, while the voids close and more homogeneous films appear with increasing $V_{b}$. The increased homogeneity is ascribed to the higher ion energies generated at higher $V_{b}$ (Paper I). The differences between $C N_{x}$ at the same $V_{b}$ films are due to the different amount of ions in each technique and the different duty cycles (Paper I). The low duty cycles of HiPIMS processes hinder the formation of homogeneous films at low $V_{b}$, although at $V_{b}=120 \mathrm{~V}$, the films become homogeneous. In the DCMS, the continuous operation of $V_{b}$ contributes to the formation of homogeneous films from $V_{b} \geq 100 \mathrm{~V}$, while in MFMS processes the high ion densities together with the high duty cycle contribute to the formation of homogeneous films from $V_{b}$ as low as $60 \mathrm{~V}$. 


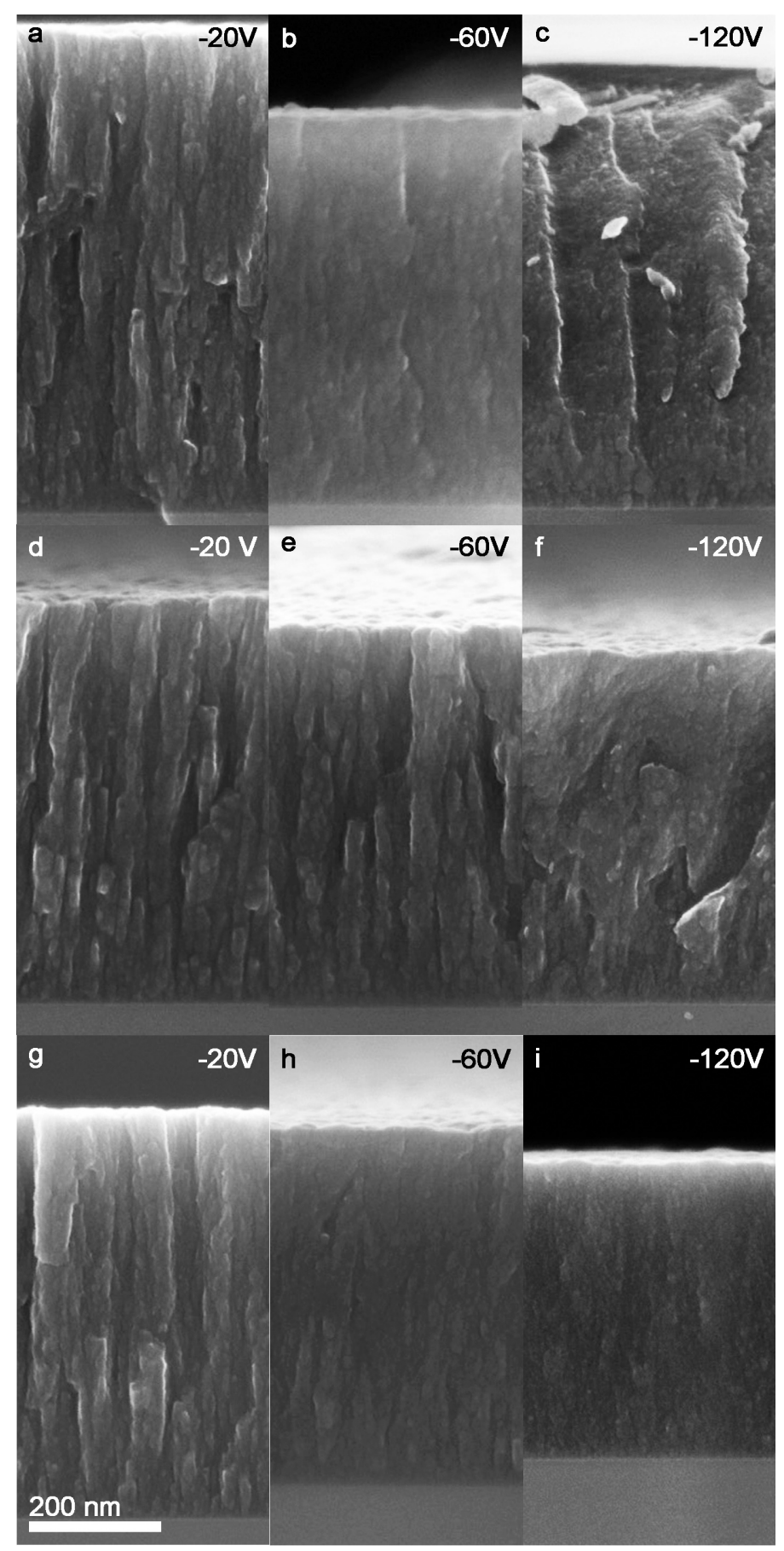

Figure 2.6: Cross-sections of $C N_{x}$ thin films deposited by (a)-(c) MFMS, (d)-(f) HiPIMS, and (g)-(i) DCMS at different $V_{b}$. 
2.3. DC AND PULSED NEGATIVE BIAS VOLTAGE 


\section{Chapter 3}

\section{Thin film characterization}

\subsection{Structural characterization}

\subsubsection{Scanning Electron Microscopy (SEM)}

The scanning electron microscope is one of the most, if not the most widely, employed instrument in thin film characterization. The relatively inexpensive as well as convenient operation compared to a transmission electron microscope (TEM), makes it a useful tool for fast structural evaluation.

Typically, electrons are thermionically emitted from a tungsten, $L a B_{6}$-cathode filament or in many modern microscopes from Schottky field-emission source and transferred to the microscope column through an anode, where they are focused by a sequence of condenser lenses into a beam [48]. Typical energies for the electron beams are between $1-50 \mathrm{keV}$. An illustration of the electron beam path is shown in Fig 3.1(a).

The electron beam impinges the sample surface and scattering events are caused by the interaction of the electrons with the sample. Fig 3.1(b) shows the interactions of electron beam with the surface of a sample and the produced particles. A spectrum of secondary electrons, backscattered electrons and auger electrons results. The most common imaging mode relies on the detection of the secondary electrons. The secondary electrons originate from the subsurface of the sample, due to their very low energy, although their small exit depth enhances the topographical resolution. The preparation of the samples for SEM includes fracture cross-sections of the film. Cross-sectional SEM is a fast way to visualize the changes in the morphology of the $C N_{x}$ films and to evaluate the changes induced in the film structure. 


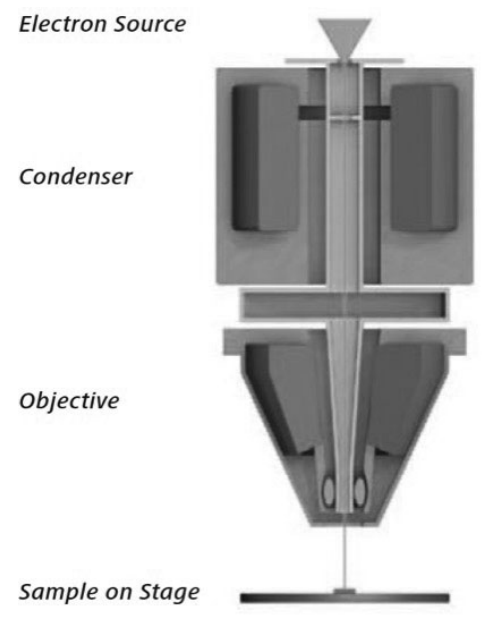

(a)

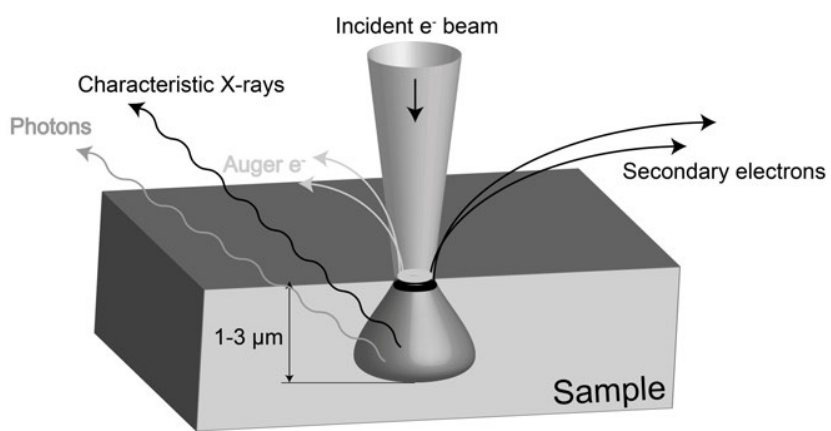

(b)

Figure 3.1: Illustration of (a) an electron source and lens sequence which the incident $e^{-}$beam passes through (illustration from www.zeiss.com) and (b) the interactions on a sample surface after the $e^{-}$beam impinges on the surface.

Moreover, the technique offers a practical method to connect the changes observed in other quantification measurements, such as the density of the films, with the resulted morphology. Figure 2.6 shows an example of SEM cross-sections of $C N_{x}$ films deposited on $S i(001)$ substrates by different magnetron sputtering techniques and at different $V_{b}$. From the cross-sections of Fig. 2.6 differences in porosity are observed through a contrast difference between denser and underdense areas. For $C N_{x}$ films deposited at $V_{b}=20 \mathrm{~V}$, clear boundaries are observed, which imply separation of material into columns. As the ion energies increase $\left(V_{b}>20 \mathrm{~V}\right)$, no clear boundaries are observed, the columns close and more homogeneous morphologies are observed.

\subsubsection{Transmission Electron Microscopy (TEM)}

TEM is rather a sophisticated and expensive, but important and powerfool instrument for visualization of atomic arrangements in materials. The first TEM was constructed by Knoll and Ruska the early 1930s [58], and along with Louis de Broglie's theory for the wave characteristics of electron [59], it decisively contributed to the development of the electron microscopy. The robustness of the TEM technique lies on the fact that it offers direct information regarding the microstructure of the films, thus it is widely used in thin film technology. The analytical resolution of a TEM can be used in different modes, such as high-resolution transmission electron mi- 
croscopy (HRTEM), scanning transmission electron microscopy (STEM) or selected area electron diffraction (SAED), while each of them serves additionally for the better understanding of the structure of a material.

TEM instrumentation and operation from the material science perspective are thoroughly treated in [60]. In a very simplified context, TEM consists of three main parts: a) an electron gun, b) the column including magnetic lenses and apertures and c) a CCD camera. Nowadays, in more expensive TEM instruments, electrons are emitted by field emission guns (FEGs), operating in the range of $60-300 \mathrm{keV}$, depending on the operation mode but also on the instrument's limitations. The energy of the electron beam for the TEM imaging and SAED acquisition in our study was set to $300 \mathrm{keV}$, in order to improve the image contrast. The electrons are transmitted in the illumination system of the microscope, which consists of a sequence of magnetic condenser lenses and apertures. The electron beam exits this sequence of lenses as coherent and parallel as possible and illuminates the sample, which is steadily placed in the sample holder (Fig. 3.2). The electron beam is transmitted through the sample, whereas some electrons are elastically or inelastically scattered upon their interaction with the sample atomic potentials. Thus, electrons with different wave functions exit the sample.

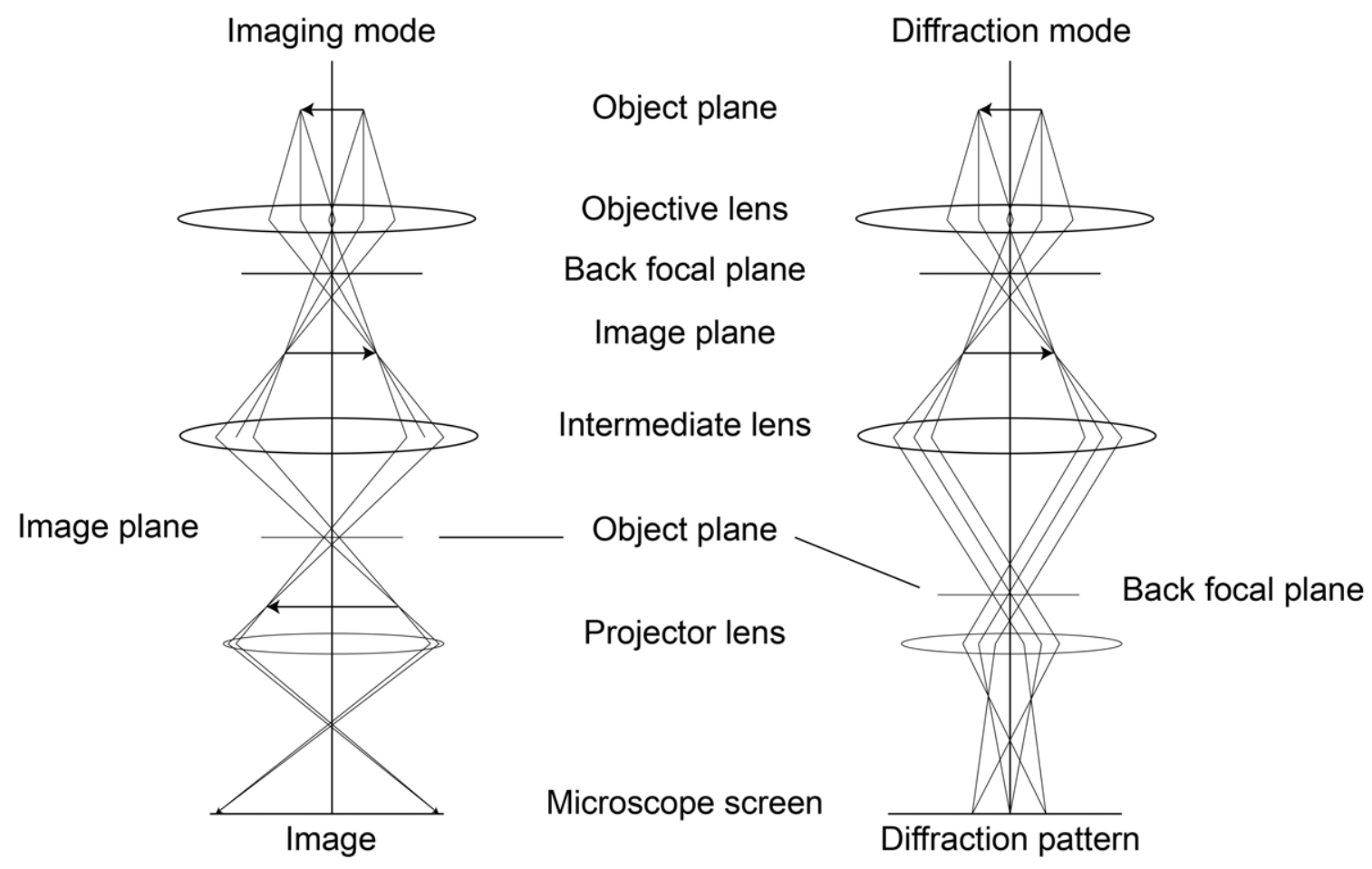

Figure 3.2: Illustration of imaging and diffraction mode of a TEM. 
A sequence of an objective and a projector lens follows under the sample holder, which are responsible for the convergence of the appropriate scattered electron beams. A pattern of the electron densities is formed and projected at the back focal plane of the objective lens. The electron beam is magnified before it results on the CCD camera. In the latter part of the electron beam route, the option between imaging mode or diffraction mode can be done, by using the respective mode. For the observation of a certain area of interest of the sample, the selected area diffraction (SAD) aperture is inserted at the image plane (Fig. 3.3) and a selected area electron diffraction (SAED) pattern is acquired.

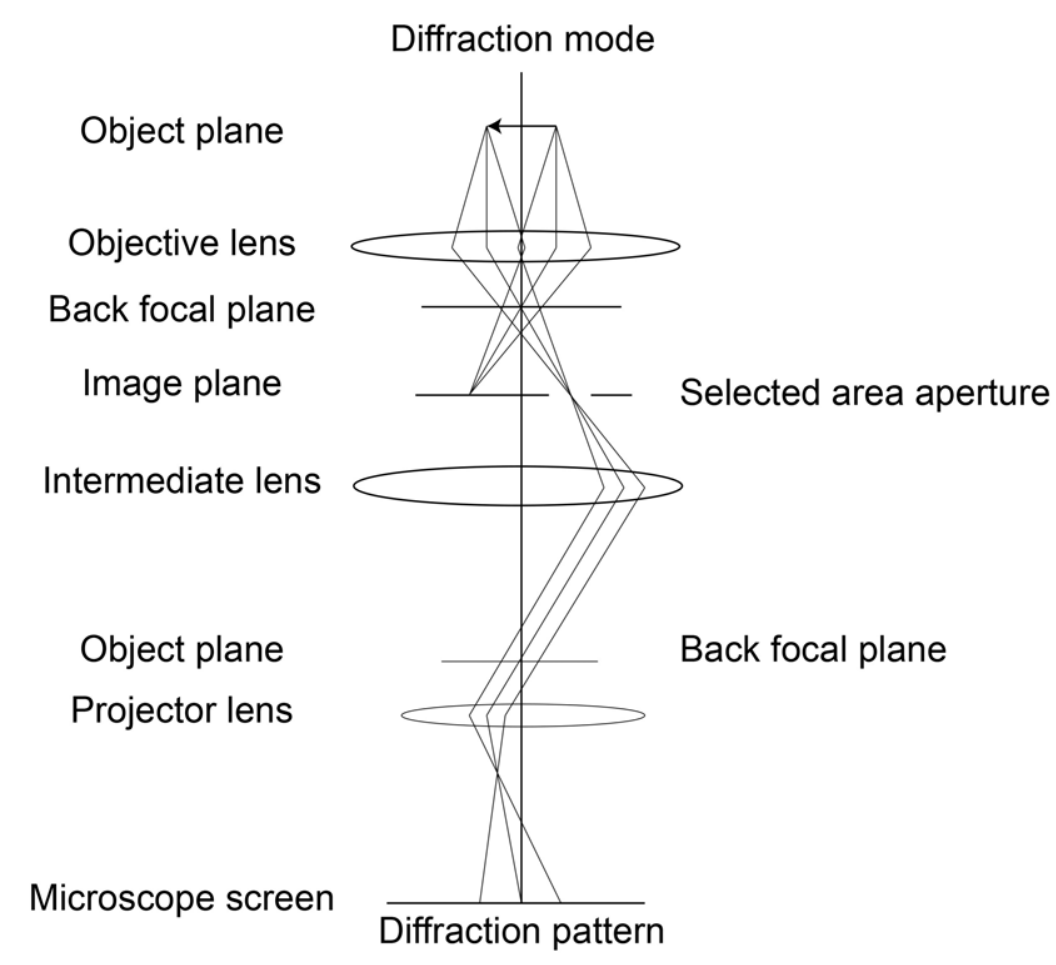

Figure 3.3: Illustration of the SAED configuration in the imaging system of a TEM.

Different kinds of diffraction patterns are obtained, according to the crystallographic microstructure of the material. Crystalline materials exhibit generally dots in a distinguishable pattern, while polycrystalline materials present rings often with spots. Amorphous materials present also rings, although broader, and without the spots observed in polycrystalline materials.

$C N_{x}$ thin films were extensively examined using HRTEM and SAED modes in the past and two main structural configurations were identified for $C N_{x}$ thin films; amorphous carbon nitride $\left(a-C N_{x}\right)$ and fullerene-like carbon nitride $\left(F L-C N_{x}\right)$ [61], [62], [63]. The observations of $C N_{x}$ films in TEM should be approached with caution. 
Cross-sectional TEM is a useful imaging mode and allows for observations of shortrange order (SRO) in the films, although in some cases, SRO may be present, but not observable in the image. This means that very small ordered nanostructures can be present in the film, but they are too small for imaging even in HRTEM. There might also be the case that the images contain the information, but the eye of the observer is not capable to resolve this information. For $C N_{x}$ films, such configurations are mainly the $F L-C N_{x}$ nanostructures, which significantly change not only the structure of the film, but also its properties, and for this reason is important to be able to resolve the film microstructure. Hence, the discrimination between $a-C N_{x}$ and $F L-C N_{x}$ is made by both imaging mode and SAED patterns. While $a-C N_{x}$ films exhibit two distinct rings at $\sim 1.2 \AA$ and $\sim 2 \AA$ correlated to amorphous microstructure, the $F L-C N_{x}$ allotrope presents one more diffraction ring at $\sim 3.5 \AA$, which closely corresponds to the one observed in graphitic diffraction patterns, indicating short-range order (SRO) [62]. Fig. 3.4 shows a TEM image with the corresponding SAED pattern of the $C N_{x}$ film deposited with MFMS at $V_{b}=120 \mathrm{~V}$. Both the image and the SAED pattern reveal the amorphous character of the film (Paper I).

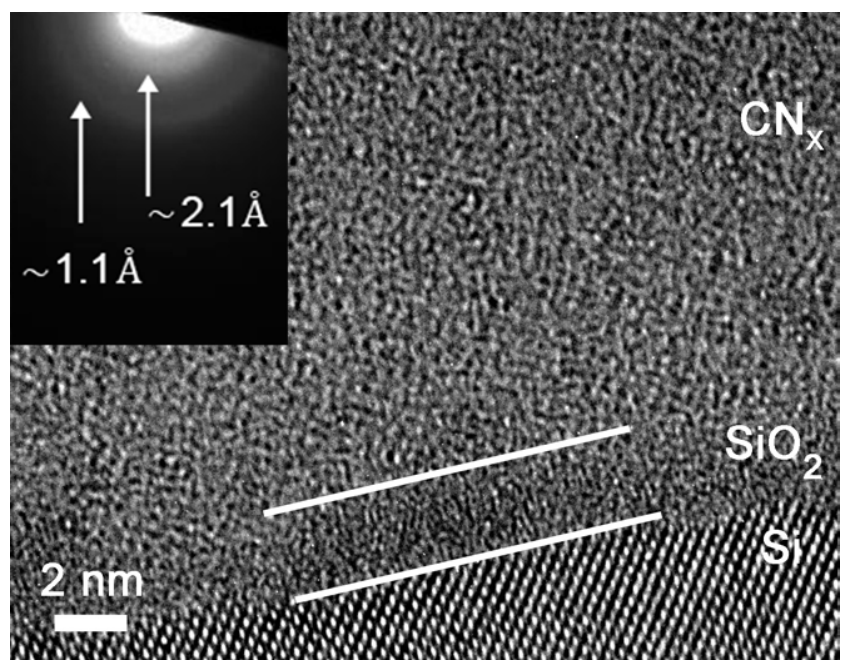

Figure 3.4: HRTEM micrograph and SAED pattern (inset) of the $C N_{x}$ film deposited at $V_{b}=120 \mathrm{~V}$ with MFMS.

In some cases though, the third ring located at $\sim 3.5 \AA$ is also difficult to observe, since it may be located in the central disk of the incident beam in the diffraction pattern. The microstructure of the $C N_{x}$ film demonstrated in Fig. 3.4 may show characteristics of SRO, where in some areas of the film small fragments of two or three bended planes formed arrays of less than $1.5 \mathrm{~nm}$. Despite that HRTEM 
and SAED are very powerful tool for the determination of SRO in $C N_{x}$ films, a complete assessment of the microstructure of these films should also include x-ray photoelectron spectroscopy, since it provides with additional information regarding the $N$ bonding configurations and whether an amorphous or $F L$ structure prevails (see also subchapter 3.1.4). Thus, the film above is characterized as amorphous.

\subsubsection{Focused Ion Beam (FIB)}

Focused ion beam is recently used for the preparation of TEM samples, especially when there is need for selection of a special area of the sample or asymmetrical substrate shapes need to be analyzed. Moreover, very small pieces (on the order of a few $\mu \mathrm{m}$ ) of the material can be cut with FIB, making possible the analysis of specific features of the samples. In thin film technology, FIB is used for the preparation of both plan views and cross-sections of the samples. FIB instruments resemble SEM, but instead of electrons, $G a$ ions are used for the imaging and milling of the sample. Two FIB configurations can be met; a) the single-beam FIB where one source is used to produce the $G a$ ions for imaging and milling and b) the dualbeam FIB in which there are two guns, usually placed with an angle of $52-54^{\circ}$, where one is an electron gun (usually FEG) and the other a $G a$ ion gun. A dual FIB configuration is presented in illustration in Fig. 3.5.

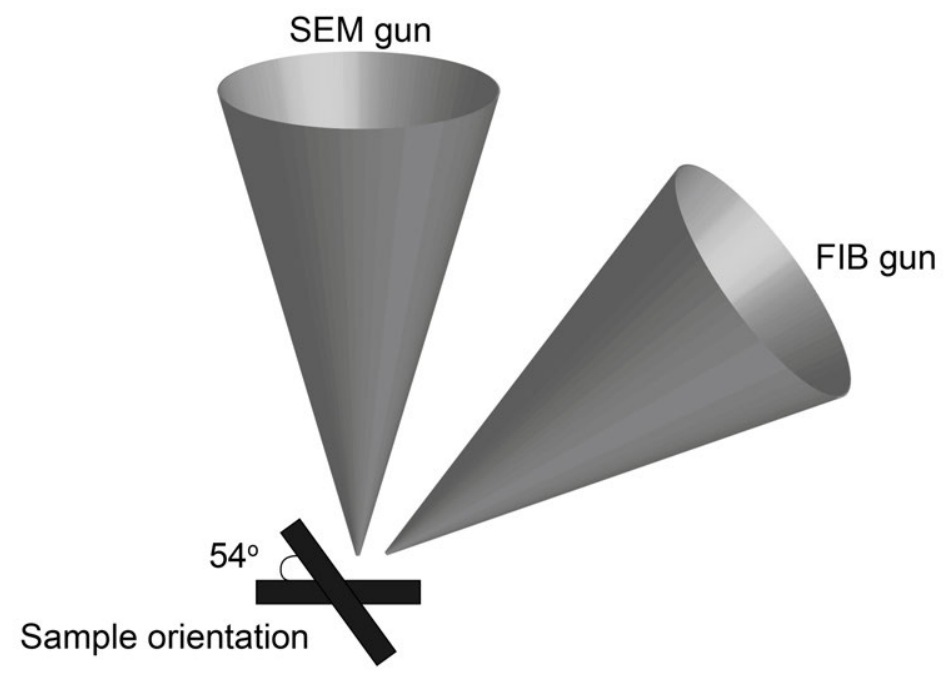

Figure 3.5: Illustration of a dual FIB configuration, with a FIB and an SEM gun.

The $C N_{x}$ thin films were proved to be sensitive to ion radiation and must be treated carefully even from the stage of TEM sample preparation [64]. Small angle cleavage technique (SACT) and conventional sample polishing followed by ion beam 
milling have been used to prepare TEM specimens. For the scope of this study, the most appropriate technique poses the focused ion beam (FIB). Steel substrates used in applications such as ball bearings are very hard for cleaving, moreover the thickness and shape of the steel substrates may vary, prohibiting essentially the use of SACT and conventional polishing. FIB offers better control of the TEM preparation stage and allows for precise selection of the area of interest, when needed as for example in analysis of different parts of wear tracks. A thin Pt layer is deposited prior to the milling in order to protect the film from the $G a$ beam. The lift-out technique is mainly used for the TEM cross-section preparation of thin films [65]. Ion energies of $30 \mathrm{kV}$ and currents from $5 \mathrm{nA}$ to $500 \mathrm{pA}$ are used for the milling of the sample, although the case of $C N_{x}$ films is an exception. Apart from $G a$ implantation, which poses problems for all kind of materials, it was reported that $C N_{x}$ microstructure, in film form, is sensitive to high energy ion milling and SRO features, such as fullerene-like, may become amorphized. Thus, the analysis of $C N_{x}$ films deposited on steel substrates of various shapes, accompanied by the identification of possible graphitic or fullerene-like phases in $C N_{x}$ films becomes complicated, if not impossible. However, the thickness of the amorphized layers due to $G a$ milling can be reduced with decreasing $G a$ ion energy. Consequently, the energy of the electron beam at $300 \mathrm{kV}$, not only improves the image contrast, but also helps to enhance the imaging of the unaffected film areas. This technique further enhances the thickness ratio of the original unaffected area of the sample to the amorphized external layer due to FIB preparation [64]. In order to improve this thickness ratio, specific steps were followed in the preparation of the TEM lamella.

The TEM cross-sections are prepared using the lift-out technique and initial milling ion energy of $30 \mathrm{kV}$, although for the final polishing of the TEM lamella, the $G a$ ion energy was reduced to $5 \mathrm{kV}$. Thus, the amorphized layer thickness was reduced to $\sim 5 \mathrm{~nm}$ and the amount of $\mathrm{Ga}$ implantation decreased significantly. The $G a$ implantation is further reduced using lower ion currents at the final stages of the polishing. During the milling with $30 \mathrm{kV}$ ion energy, currents of $2 n A, 1 n A$, $500 p A, 200 p A, 100 p A$ and $20 p A$ were sequentially used for the preparation of the lamella, in order to decrease the possibility of $G a$ implantation. At the final stage of the polishing of the TEM lamella, a current of $100 p A$ was used, which was the lowest possible current reached. Fig. 3.6 shows a comparison between a $C N_{x}$ film prepared by FIB using $30 \mathrm{kV}$ and a $C N_{x}$ film prepared using $5 \mathrm{kV} G a$ ion energy. In the case where $30 \mathrm{kV}$ was chosen, the density of implanted $G a$ areas 
is presented higher compared to the density of respective areas in the case of $5 \mathrm{kV}$ $G a$ ion energy. In the case that $5 k V G a$ ion energy was selected, the implanted $\mathrm{Ga}$ appears to affect areas with a diameter less than $2 \mathrm{~nm}$, while such areas are much larger in the case of $30 \mathrm{kV}$ (>2nm in some cases). Especially, at thin parts of the film, such as the edges, higher amount of Ga implantation is observed and an interpretation of film structure is not possible. Thus, the obtained final thickness of the TEM lamella should be above a critical thickness, below of which the amount of implanted $G a$ makes the observation of the film microstructure impossible.

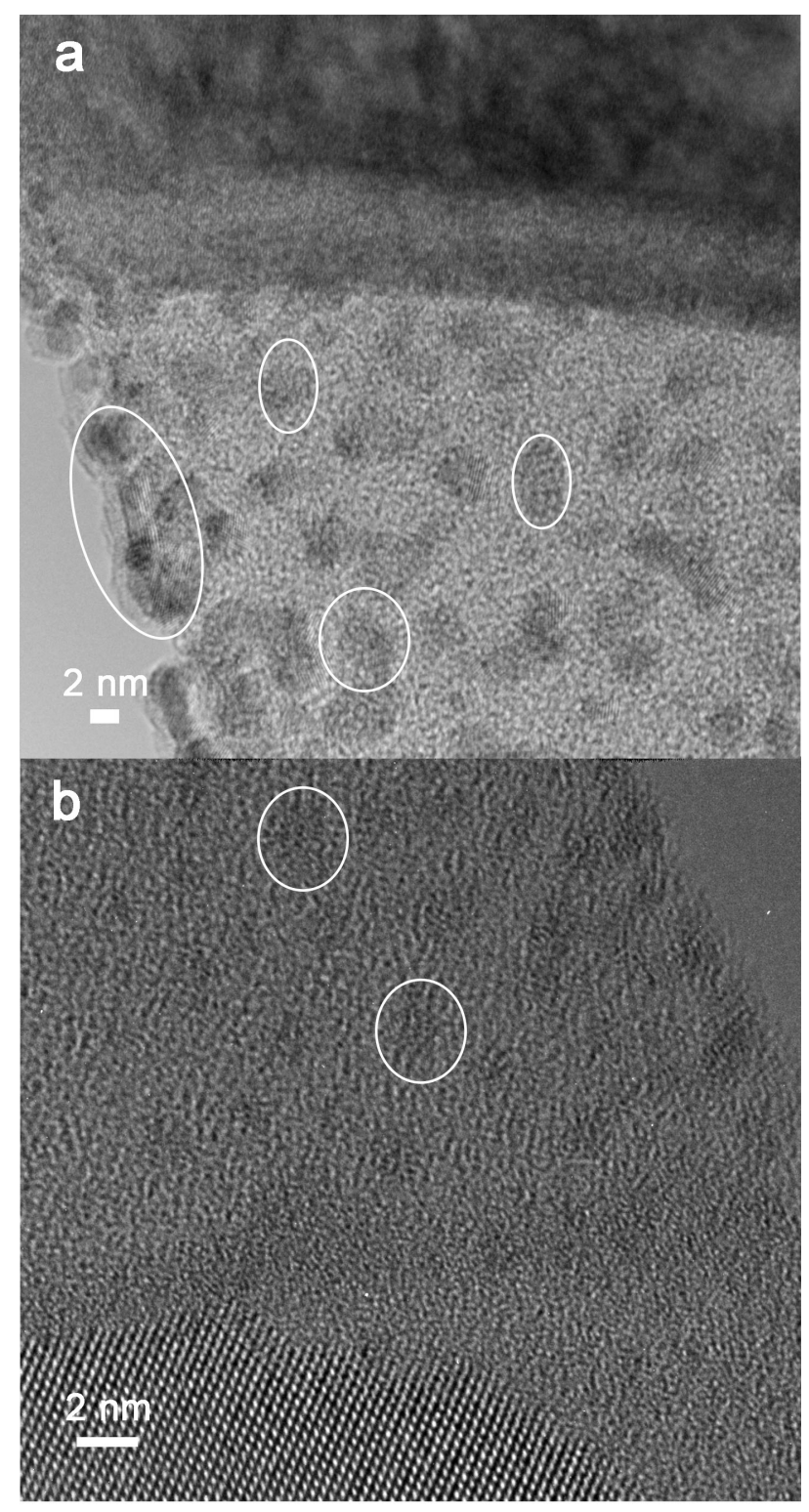

Figure 3.6: TEM cross-sections of a $C N_{x}$ thin film, prepared using (a) $30 \mathrm{kV}$ and (b) $5 \mathrm{kV} G a$ ion energy. 


\subsubsection{X-ray Photoelectron Spectroscopy (XPS)}

XPS is a surface analytical characterization technique, extensively used in the past for the determination of the bonding type in $a-C$ and $a-C N_{x}$ films [44], [43], [36], [66], [67], [61], [20], [68], [69], [38]. The technique relies on the photoelectric effect i.e., emission of electrons from a solid surface upon irradiation with photons of sufficient high energy. The probing depth of XPS is 3-10 $\mathrm{nm}$ and the technique provides useful information regarding the $N$ bonding in the $C$ matrix, extracted from C1s and N1s core level spectra.

For XPS measurements, the samples are placed in ultra-high vacuum and irradiated with photons with energies in the X-rays range. Commonly $\mathrm{Mg} K_{\alpha}=1253.6$ $\mathrm{eV}$ or $\mathrm{Al} K_{\alpha}=1486.7 \mathrm{eV}$ sources are used. Due to photoelectric effect, electrons are emitted and if they are generated near the surface, they can escape into the vacuum chamber.

The binding energy, $E_{B}$, of the electron is deduced using the Einstein's formula:

$$
E_{B}=h \nu-\phi_{\text {spectr }}-K E
$$

where $h \nu$ is the photon energy, $E_{B}$ is the binding energy of the core electron, $\phi_{\text {spectr }}$ is the work function of the spectrometer and $K E$ is the kinetic energy with respect to the Fermi level.

XPS is particularly useful to obtain information about concentration of $C, N$, and $O$ in $C N_{x}$ films, as well as give insights into the $C$ - $N$ bonding configuration. The deconvolution of $\mathrm{C} 1 \mathrm{~s}$ core level spectra is a difficult task, since no theoretical background is established for $C N_{x}$ films. There is a large number of publications, where authors assign all peaks to $s p^{2}$ or $s p^{3}$ bonding states, ignoring that $N$ incorporation can induce either $s p^{2}, s p^{3}$ or $s p$ bonds, altering significantly the bonding configuration of the $C$ matrix and that $C N_{x}$ films are $s p^{2}$-rich materials exactly due to that $N$ incorporation. Different components can contribute to the C1s core level spectra, due to the presence of $C-N$ bonds. The peak model of C1s and N1s core level spectra of $C N_{x}$ thin films used in our study is presented in Fig. 3.7. C1s core level spectra of $C N_{x}$ films can be satisfactorily fitted by 5 components. The components at $\sim 284.7 \mathrm{eV}(C 1)$ and $\sim 286 \mathrm{eV}(C 2)$, also observed in $N$-free $D L C$ films, are assigned to $C s p^{2}$ and $C s p^{3}$ hybridized states. The component at the binding energy of $\sim 287.3 \mathrm{eV}$ is due to $C$ - $N$ bonds (C3), although no discrimination between $s p^{2}$ or $s p^{3}$ bonding states can be done. This is due to the different possi- 
bilities for $s p^{2}$ or $s p^{3}$ bonds that $N$ can form upon its incorporation in the films. Thus, a resolution into $s p^{2}$ or $s p^{3}$ configuration can not be carried out, since $C 3$ component is an intermixing of $C-N s p^{2}$ and $s p^{3}$ bonds. $C 1$ and $C 2$ components may also be influenced by $N$ presence, since $N$ can also form $s p^{2}$ and $s p^{3}$ bonding configurations with $C$. For accurate interpretation of $s p^{2}$ and $s p^{3}$ contents in $C N_{x}$ films, more characterization techniques should be involved in combination with XPS, such as magnetic resonance spectroscopy and/or electron energy loss spectroscopy. In the same binding energy region, contributions due to $C-O(C 4)$ and $C=O(C 5)$ bonds appear (at $\sim 289 \mathrm{eV}$ and $\sim 290.7 \mathrm{eV}$, respectively), depending on the $O$ contamination in the films.

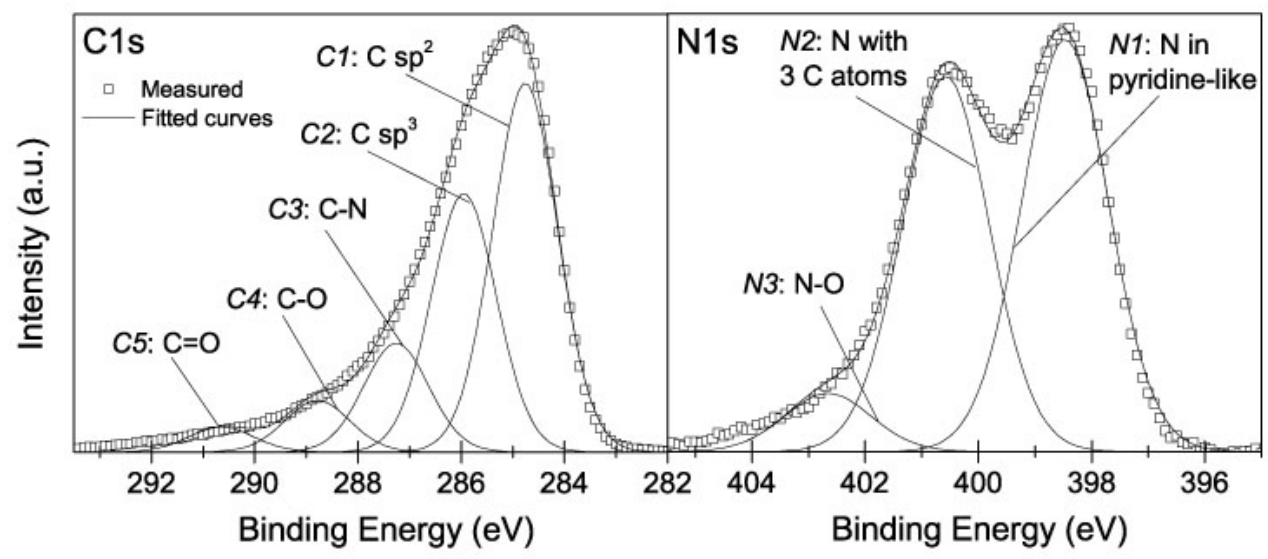

Figure 3.7: XPS peak model for the deconvolution of C1s and N1s core level spectra of the film deposited by MFMS at $V_{b}=120 \mathrm{~V}$.

N1s core level spectra show three contributions; $N 1$ at $\sim 398.6 \pm 0.2 \mathrm{eV}$, attributed to $N$ bond in 2-fold coordination at the periphery of graphene sheets in the $C$ network (the pyridine-like structure), $N 2$ at $\sim 400.6 \pm 0.1 \mathrm{eV}$, attributed to $s p^{2}$ hybridized $N$ bond to three $C$ atoms in a graphitic network and $N 3$ at $\sim 402.7 \pm 0.1$ $e V$ attiributed to $N-O$ bond. $N 2 / N 1$ peak area ratio and the separation between $N 1$ and $N 2$ peaks in N1s core level spectra are characteristic quantities which can reveal either the amorphous or the fullerene-like structure of the films [20]. Peak area ratios of $N 2 / N 1>1$ with a N1-N2 separation of $\geq 2 \mathrm{eV}$ denote fullerene-like microstructure. The low growth temperatures used in our study favor the $a-C N_{x}$ films. N1s core level spectra reveal small differences between films deposited with different $V_{b}$. Increasing $V_{b}, N 2 / N 1$ peak area ratio increases for films deposited by MFMS. Hence, $N$ presents a preference in bonding in a configuration with three $C$ atoms rather than in pyridine-like configuration. $N 2 / N 1$ peak area ratio for films 
deposited by HiPIMS and DCMS does not show significant difference and dependency on $V_{b}$.

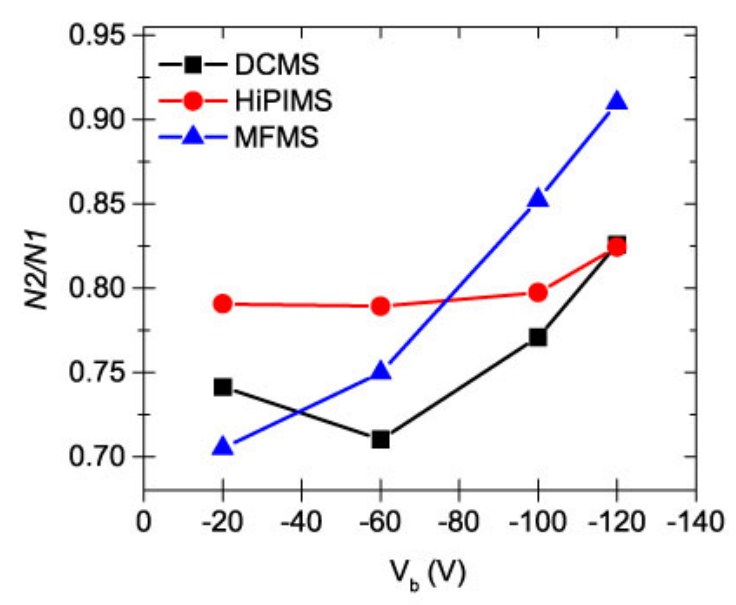

Figure 3.8: $N 2 / N 1$ peak area ratio of $C N_{x}$ films deposited by MFMS (triangles), HiPIMS (circles), and DCMS (squares) as a function of $V_{b}$.

In the above interpretations regarding the bonding configurations in the films for all deposition modes, it is assumed a priori that $N$ is bonded uniformly in the $C$ matrix, and that the $s p^{2}$ and $s p^{3}$ content in the films is also uniformly distributed. There is no evidence for this though, since the probing depth of XPS is rather small in comparison with the film thickness, but a depth profile would give a sense of the distribution of $N$ incorporation.

\subsubsection{X-ray Reflectivity (XRR)}

$\mathrm{XRR}$ is a relatively fast and non-destructive technique for the determination of density, thickness, and roughness of thin films and also provides information about the layer periodicity of multilayered thin films. The refractive index in solids is slightly smaller than unity for x-rays and total external reflection occurs at low angles of incidence. The x-rays will start to penetrate into the film, as the incident angle increases above a critical angle $\theta_{c}$. For a structure with one layer of film deposited on a bulk substrate, there are two different refractive indexes; $n_{1}$ for the top thin layer and $n_{2}$ for the bulk substrate. Thus, the reflection of x-rays at the interface of layers with different refractive indexes will cause interference of the reflected beams. Information regarding the thickness and the roughness of the films can be extracted, although for $a-C$ and $a-C N_{x}$ films, thickness or roughness of the films is not always accessible from the XRR profiles. 
Density of both $a-C$ and $a-C N_{x}$, though is obtained accurately and only from the critical angle. Critical angle, $\theta_{c}$, is proportional to the electron density, hence the mass density of the material. The density of $a-C N_{x}$ can be estimated by neglecting contributions from $N$ content, even if $N$ is present in the films. For $C$ films with density very close to the density of the substrate (i.e., $S i$ in most of the cases), a simulation of the reflectivity is needed to extract the density of the film, because the presence of the film acts only as a perturbation of the shape of critical angle. Fig. 3.9 shows a respective fitting, obtained for the film deposited by HiPIMS at $V_{b}=100 \mathrm{~V}$.

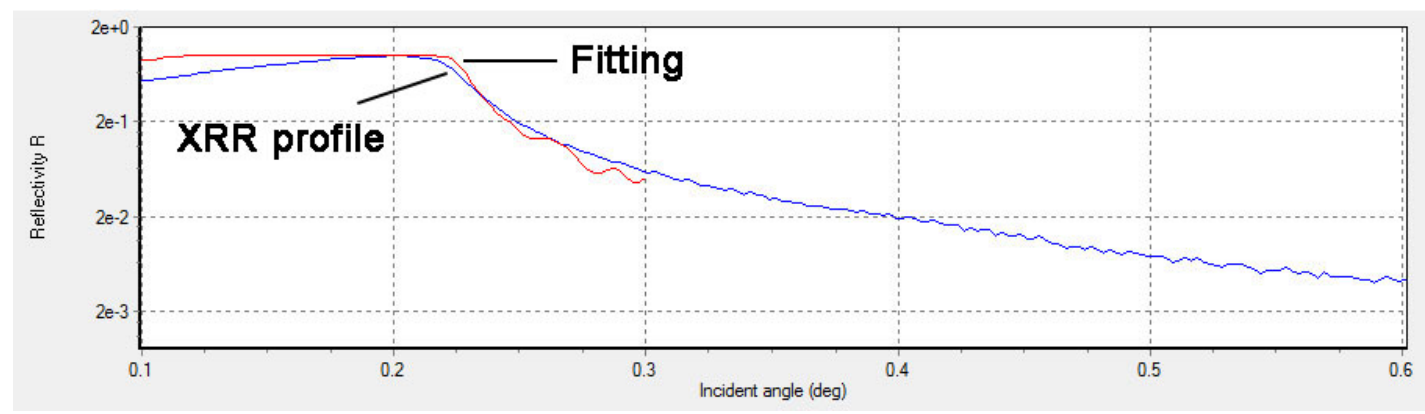

Figure 3.9: XRR profile and the respective fitting for the film grown by HiPIMS at $V_{b}=100 \mathrm{~V}$.

In the case of $C$ films with very low density (porous carbons or $a-C$ ), a double critical angle is distinguishable and it allows for a direct determination of film density. If the $C$ film is denser than the substrate (i.e., $S i$ ), such as in the case of $t a-C$, only a single critical angle is observed, which gives the density of the carbon films.

DC magnetron sputtered $a-C N_{x}$ films exhibited generally densities lower or close to that of graphite [37], [22] and sputtered $C N_{x}$ [21]. Films produced by MFMS, HiPIMS, as well as DCMS in this study, exhibit also densities lower and close to that of graphite and sputtered $C N_{x}$. A dependency on $V_{b}$ is also observed, where density of all films presents a linear increase with increasing $V_{b}$ (Fig. 3.10). These films densify because higher $V_{b}$ values give more momentum to the incident ion species that results in enhanced adspieces mobility to find higher bonding-coordination (energy) sites and also recoil implantation, which effectively closes the intercolumnar porosity, if not intercalates the forming structure by $N$ or $A r$ (Paper I). 


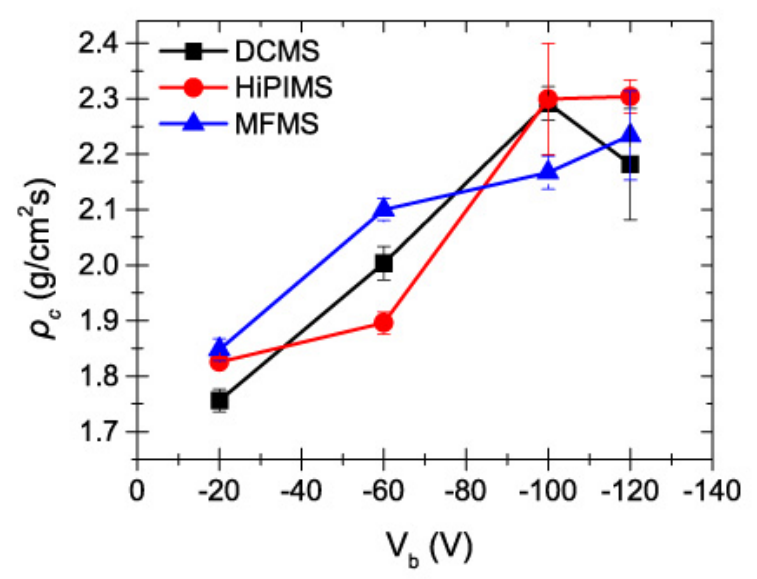

Figure 3.10: Density of $C N_{x}$ films deposited by MFMS (triangles), HiPIMS (circles) and DCMS (squares) as a function of $V_{b}$.

\subsection{Mechanical characterization}

\subsubsection{Profilometry}

Profilometry is a scanning technique used for thickness or roughness measurements of thin or thick film surfaces. During a profile acquisition, a stylus traces the topography of a film-substrate step, recording the thickness of the film. The roughness of the films is also measured as surface variations, where the surface variations are recorded electromechanically with a stylus.

Profilometry can also be used for residual stress measurements of films. The possibilities for residual stress measurements in amorphous films are limited, exactly due to the lack of crystalline or at least pollycrystalline order. This is also valid for $F L$ films. Thus, profilometry is a very fast and useful technique for obtaining residual stress of amorphous thin films, therefore of $a-C$ and $a-C N_{x}$ thin films also. The radius of curvature of a coated substrate is measured and the residual stress is calculated by the modified Stoney's equation for thin films:

$$
\sigma_{f i l m}=\frac{Y_{\text {sub }} d_{\text {sub }}^{2}}{6 R\left(1-\nu_{\text {sub }}\right) d_{f i l m}},
$$

where $\sigma_{f i l m}$ is the residual stress of the film, $d_{\text {sub }}$ is the thickness of the substrate, $\nu_{s u b}$ is the Poisson's ratio of the substrate, $Y_{s u b}$ is the Young's modulus of the substrate (for Si substrates: $d_{S i}=525 \mu \mathrm{m}, \nu_{S i}=0.36$ and $Y_{\text {sub }}=169 \mathrm{GPa}$ [70]), $d_{\text {film }}$ is the thickness of the film and $R$ is the radius of curvature of the sample 
as measured with the stylus profilometer. The radius of curvature of an uncoated substrate is measured before the measurements of the coated substrates. Eventually, the residual stress of the uncoated substrate is subtracted from the residual stress of the coated substrate, in order to extract the stress induced by the film. Fig. 3.11 shows the residual stress induced by $C N_{x}$ thin films deposited on $\operatorname{Si}(001)$ substrates by MFMS, HiPIMS, and DCMS, as a function of $V_{b}$.

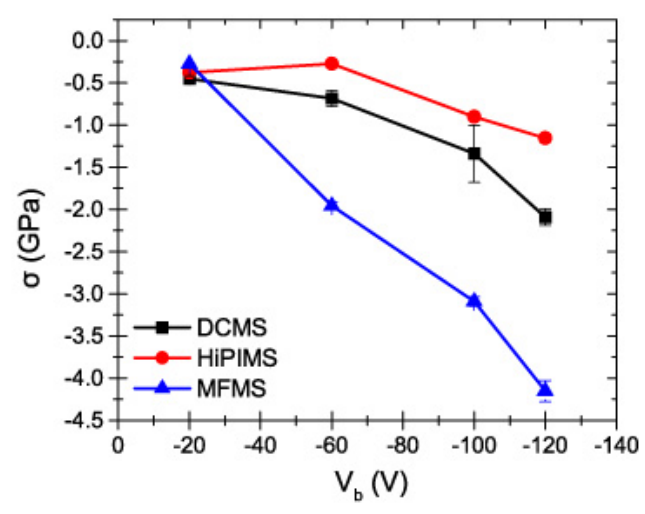

Figure 3.11: Residual stress of $C N_{x}$ films deposited by MFMS (triangles), HiPIMS (circles), and DCMS (squares) as a function of $V_{b}$.

A strong dependency of $\sigma$ on $V_{b}$ is observed for $C N_{x}$ films grown with all magnetron sputtering techniques, moreover $\sigma$ at each technique is influenced to a different extent by $V_{b}$. The increasing $\sigma$ with increasing $V_{b}$ are primarily due to higher energy bombardment, which leads to suppression of the columnar growth through reduction in the formation of voids (Paper I). A dependency on Ar content with increasing $\sigma$ is also observed, where films with higher amount of intercalated $A r$ present also higher stresses. The role of working gas entrapment in the residual stress evolution remains a controversial issue in the literature [71]. In the case of the $C N_{x}$ films of this study, the forward sputtering model accounts for the $\sigma$ evolution, which states that the main reason for increased stresses is the increased ion energies [71]. Ar incorporation influences in a smaller extent the $\sigma$ evolution, atlhough it is not possible to assess how large is this influence. Nevertheless, intercalated $A r$ is expected to also contribute to the compressive stresses, primarily due to the size difference between $A r$ radius and $C-C$ and $C-N$ bond lengths, leading to a spatial distortion of the $C$ matrix. 


\subsubsection{Nanoindentation}

Nanoindentation is a method of measuring the mechanical response of thin films. During a nanoindentation, a tip of known geometry touches the surface of the film and the elastic and plastic deformation of the material are measured in terms of hardness, $H$, and elastic modulus, $E_{r}$. In the case of thin films, with thicknesses $<1 \mu \mathrm{m}$, the depth of penetration of the tip should be taken into consideration. If the penetration of the tip is deep inside the film, the substrate may influence the measurement and there is the danger to also probe the properties of the substrate, hindering the real mechanical response of the film. Thus, Berkovich tips are chosen for shallow indentations in thin films, due to their geometry, which gives higher contact areas with the film. The penetration depth is kept $<10 \%$ of the film thickness as an additional safety measure in order to eliminate substrate effects. For $C$-based (especially $C N_{x}$ ) films, the very high elasticity may consist a problem, since the penetration depth of the tip can extend the film thickness and influences from the substrate appear. Thus, one should be careful during the interpretation of the mechanical response of the films from nanoindentation curves.

For nanoindentation measurements, a Triboindenter TI 950 from Hysitron was used, employing a three plate capacitive transducer, that is used both as the actuator and sensor of the instrument. The force is applied electrostatically, while the displacement is simultaneously measured by the change in capacitance. To apply a force, a large DC bias (up to $600 \mathrm{~V}$ ) is applied to the bottom plate of the capacitor. This will create an electrostatic attraction between the centre plate and the bottom plate, which will pull the centre plate down. The force can be calculated from the magnitude of the applied voltage. The maximum normal force available from the standard transducer is approximately $10 \mathrm{mN}$.

The geometry of an indentation, using a Berkovich indenter with a vertical load $\mathrm{P}$ is demonstrated in Fig. 3.12(a), where the elastic and plastic deformation at maximum load $\left(h_{t}\right)$ and the part of the elastic deformation that recovers $\left(h_{e}\right)$ are shown. The radius of the circular impression, $\alpha$, the displacement of the surface at the perimeter of the contact, $h_{s}$, the final depth of the residual hardness impression, $h_{f}$, and the residual depth after unloading, $h_{c}$ are also denoted.

Load versus displacement curves are obtained from nanoindentation (Fig. 3.12(b)). According to the nanoindentation curves (shape and penetration depth), there are no substrate effects in the measurements, hence the hardness is extracted by: 


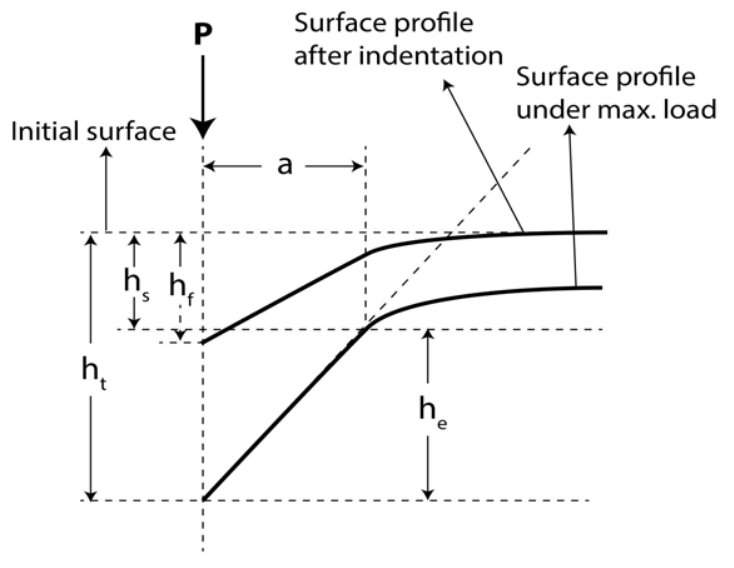

(a)

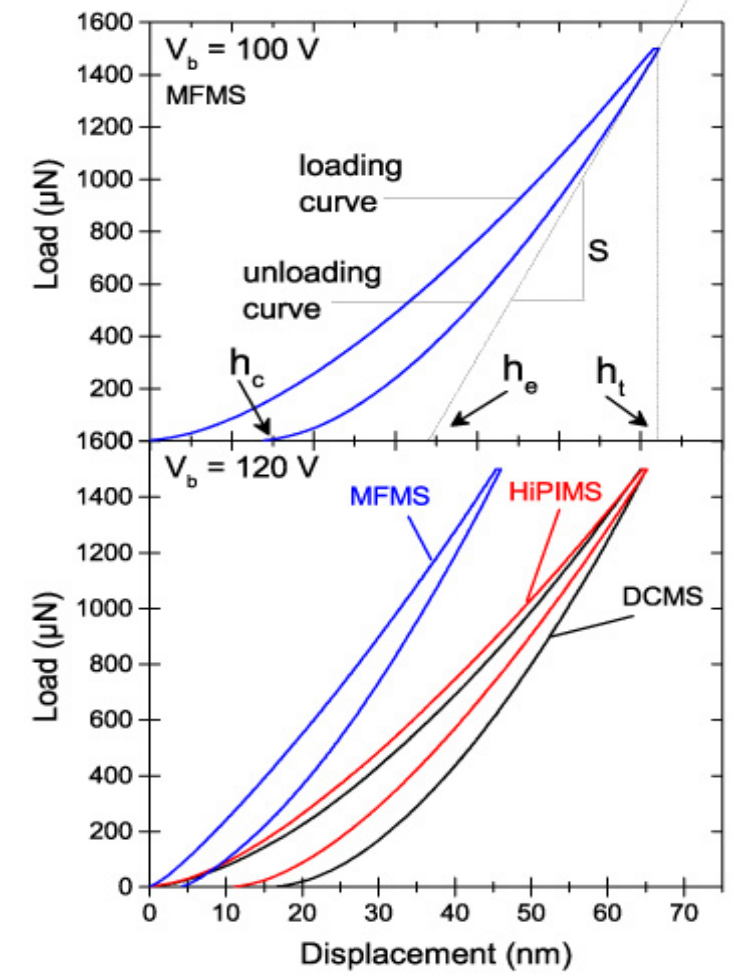

(b)

Figure 3.12: (a) Illustration of the geometry before nanoindentation and the imprint of the contact area after the nanoindentation (adopted from [72]). (b) Upper graph: an analysis of a load - displacement curve of the $C N_{x}$ film deposited by MFMS at $V_{b}=100 \mathrm{~V}$ and (b) lower graph: load - displacement curves of $C N_{x}$ film deposited by MFMS, HiPIMS and DCMS deposited at $V_{b}=120 \mathrm{~V}$, as obtained after nanoindentation.

$$
H=\frac{P}{A}
$$

where $P$ is the contact pressure and $A$ the contact area of the tip with the surface of the film. The contact area should be calculated in order to determine the effective real contact of the tip with the surface. This calibration is done before the nanoindentation measurements and is called tip area function calibration. The approach from Oliver and Pharr [72] for nanoindentation with Berkovich tip on fused silica is used in order to determine the tip area function using an appropriate fitting for the relationship: 


$$
A\left(h_{c}\right)=24.5 h_{c}^{2}+C_{1} h_{c}^{1}+C_{2} h_{c}^{1 / 2}+C_{3} h_{c}^{1 / 4}+\ldots+C_{8} h_{c}^{1 / 128},
$$

where $C_{1}$ through $C_{8}$ are constants.

An example of a load - displacement curve of an $a-C N_{x}$ thin film deposited by MFMS is shown in Fig. 3.12. With nanoindentation method is possible to extract also the reduced elastic modulus of thin films, given the contact area, which is given by:

$$
E_{r}=\frac{\sqrt{\pi}}{2} \frac{S}{\sqrt{A}}
$$

where $E_{r}$ is the reduced elastic modulus, $S$ is the contact stiffness and $A$ the contact area. The elastic recovery, ER, can be obtained as

$$
E R=\frac{h_{t}-h c}{h_{t}} 100 \%
$$

where $h_{t}$ is the maximum indentation depth and $h_{c}$ the residual depth after unloading (Fig. 3.12).

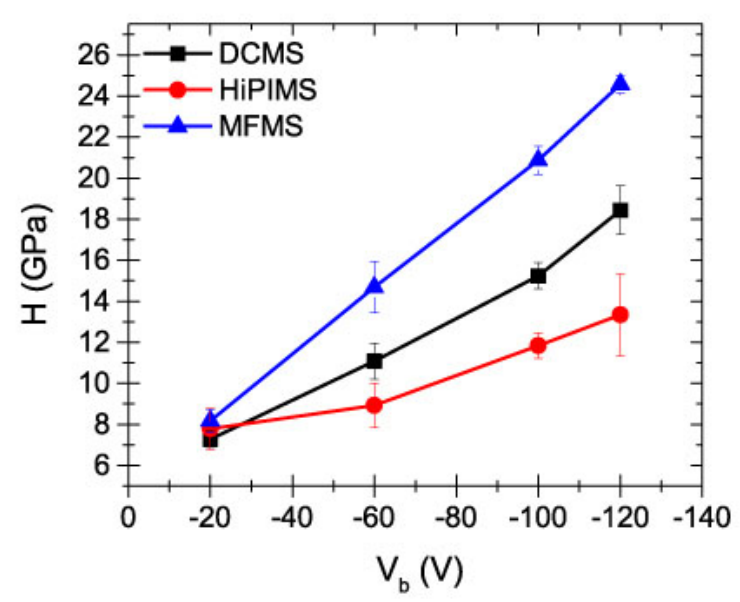

Figure 3.13: Hardness of $C N_{x}$ films deposited by MFMS (triangles), HiPIMS (circles) and DCMS (squares) as a function of $V_{b}$.

The $H$ and $E_{r}$ of $a-C N_{x}$ present remarkable dependency on $V_{s}$ (Fig. 3.13). For higher $V_{s}$, the films are presented harder and more elastic, a consequence of the densification of the $a-C N_{x}$ films produced at higher $V_{s}$, caused by higher energetic 
bombardment of the substrates. MFMS produces the harder films deposited at each $V_{s}$, while the least hard films are produced by HiPIMS at each $V_{s}$. Films produced by DCMS present intermediate values between the values obtained for $a-C N_{x}$ films deposited by MFMS and HiPIMS.

According to their $H / E$ ratio, all $a-C N_{x}$ films are proved very resilient demonstrating $H / E \geq 0.1$ (Paper I). The highest $H / E$ ratio of 0.14 is achieved by $a-C N_{x}$ deposited by MFMS and DCMS at $V_{s} \geq 100 \mathrm{~V}$. It is noted that $N$-free DLC films are classified to very resilient materials with $H / E=0.1$ [8].

\subsection{Tribological characterization}

\subsubsection{Reciprocal friction and wear test}

Reciprocal tests are used in order to assess the tribological performance of a surface, determining quantities such as friction and worn material under certain experimental conditions. The reciprocal movement of a tip over the surface, under appropriate applied load, causes changes on the surface. It also offers a good way to evaluate the tribological performance of a thin film. Depending on the applied load and the dimension of the counterpart (tip or component), the changes can be perceived at the macroscale, microscale or nanoscale. Thin films though suffer from poor adhesion on substrates and the evaluation at the macroscale is usually unsuccessful due to delamination of film from the substrate from the beginning of the test, because films are subjected to relatively high loads. A way to evaluate the tribological performance of thin films is to test them at different scale, considering always the pressure and Hertzian contacts. The evaluation of the tribological properties of films at the microscale or nanoscale is controlled by different parameters than at the macroscale, making the assessment of the results different. For instance, parameters such as humidity, surface roughness or contamination of the surface affects with different ways the measurements at the micro and nanoscale than at the macroscale. Moreover, for carbon-based thin films, which exhibit low friction and wear at macroscale, measurements at the micro or nanoscale could give some better insight to the friction and wear mechanisms of these films.

It is of great value to determine the tribological response of $C N_{x}$ thin films as a function of the number of cycles, in order to assess both the durability of the films and their lifetime. Thus, a multicycle reciprocal wear test comprising 31 cycles is used in order to evaluate the tribological properties of the $a-C N_{x}$ thin films. 
Triboindenter TI 950 is used for this test with a transducer assembly having the potential to apply both normal and lateral forces (2D transducer configuration). This assembly has two additional transducer sensors mounted on opposite sides of the first, at a $90^{\circ}$ angle.

A diamond conical tip of $\sim 5 \mu \mathrm{m}$ radius of curvature and $60^{\circ}$ included angle was reciprocated under a load of $5 \mathrm{mN}$. The profile track length was set to $10 \mu \mathrm{m}$, and the wear track to $5 \mu \mathrm{m}$. Each test comprised 31 cycles, where one cycle is considered to be one forward and one backward movement of the tip (Fig. 3.14). The friction force, $F_{F}$, of each cycle was recorded. The profile of the worn surface of the films was also recorded after each wear cycle as the normal displacement versus the lateral displacement of the tip.

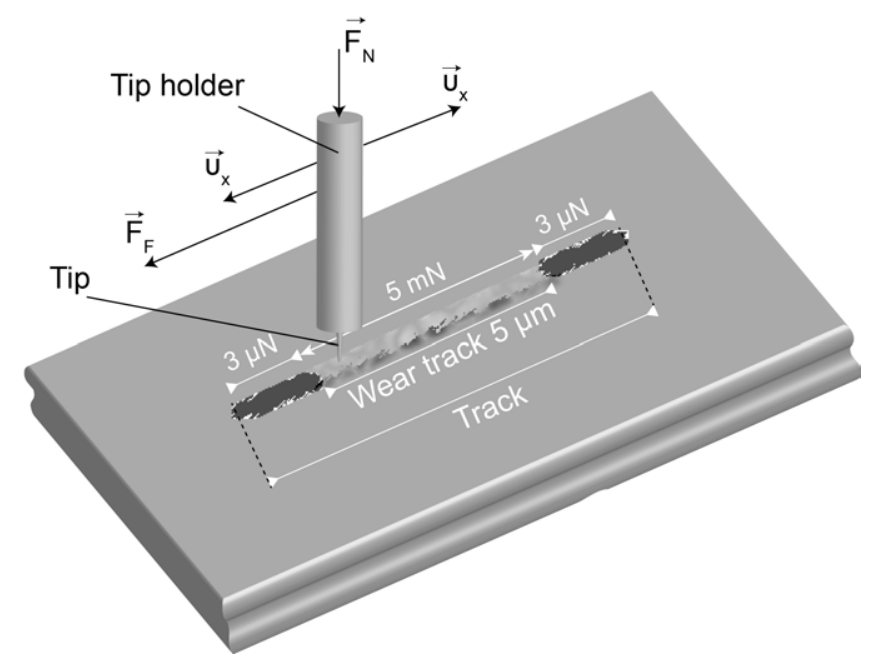

Figure 3.14: Illustration of the reciprocal wear test.

The worn material, expressed by the wear coefficient, $k$, was calculated as the integral between two measured surface profiles of a cycle, using Archard's equation [73]. The friction coefficient, $\mu$, was calculated using the relationship $\mu=\frac{F_{F}}{L}$, where $F_{F}$ is the friction force and $L$ is the applied load. Thus, the friction coefficient of each cycle is an average value obtained from the different values of $F_{F}$ during the cycle. Despite that the length of the wear track was $5 \mu \mathrm{m}$, only the data within the central zone of $4 \mu \mathrm{m}$ were used to evaluate $\mu$ in order to avoid spurious contributions due to accumulation of material in the track borders. The recorded track profiles of the $C N_{x}$ deposited with MFMS, HiPIMS and DCMS are presented with details in Paper II.

$a-C N_{x}$ thin films exhibit in many cases remarkable tribological properties. Low friction coefficients were recorded for $a-C N_{x}$ deposited either with MFMS, HiPIMS 
or DCMS and friction does not seem to depend on the deposition conditions (Fig. $3.15)$.

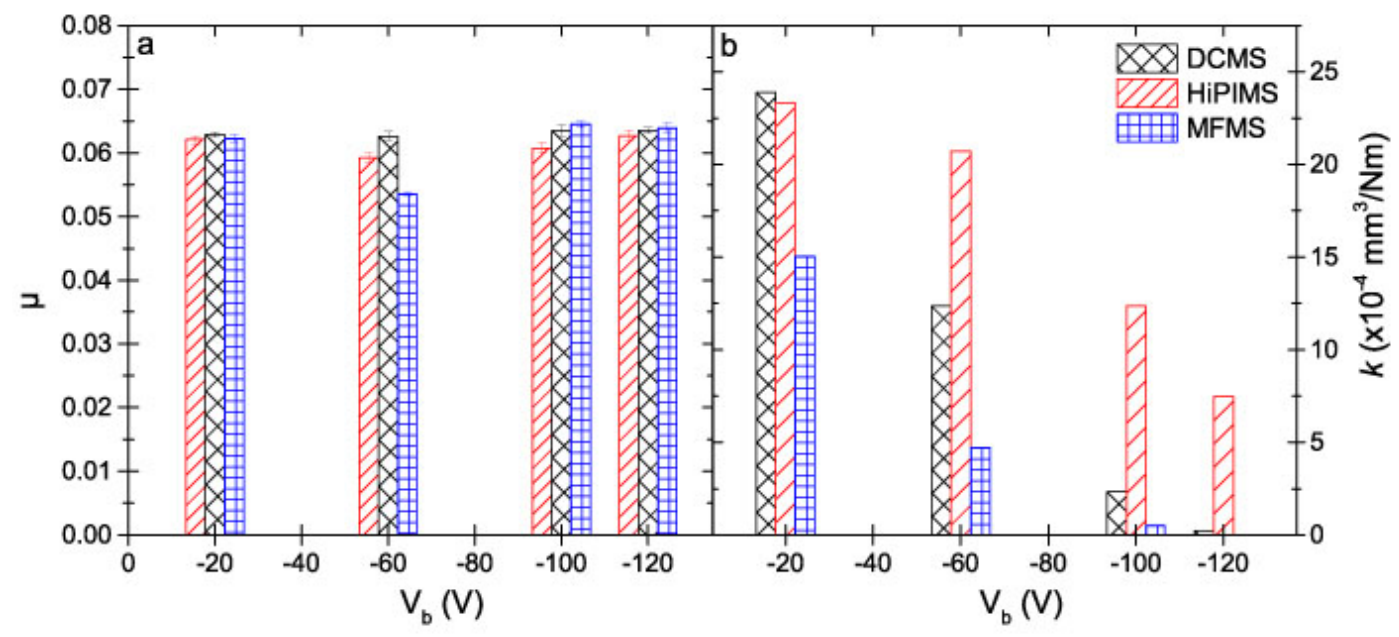

Figure 3.15: (a) Friction coefficient and (b) wear coefficient of $C N_{x}$ film deposited by MFMS (square pattern), HiPIMS (line pattern), and DCMS (cross pattern) as a function of $V_{b}$.

Friction coefficient does not show any dependency on $V_{b}$, although it depends on other parameters connected to the surface of the films, as demonstrated in Paper II, such as track roughness. Wear coefficient of the films, as obtained from the last cycle of the wear test for each $C N_{x}$ film, depends on $V_{b}$. Therefore, the morphology and the mechanical properties of the films (i.e., hardness and elastic recovery) are detrimental for the wear resistance of the $C N_{x}$ films. The combination of highly homogeneous and dense films, with high hardness and elastic recovery presents higher wear resistance. In Fig. 3.16, friction coefficient as derived from the first cycle of the wear test of the films deposited in MFMS mode as a function of $V_{b}$ is shown. Fig. 3.16 essentially shows that $\mu$ depends on the surface roughness of the track. The roughness of the films before the $1^{\text {st }}$ cycle is also presented as a function of the $V_{b}$ and it shows good agreement with the results from friction. There is no dependency of roughness with increasing $V_{b}$, although there is a trend observed between roughness and friction coefficient. It seems that when initial roughness shows relatively low values, friction coefficient is increased and as roughness increases, friction coefficient decreases. It has been demonstrated (see appendix of Paper II), that large asperities on the surface of the films, in the wear track, influence the movement of the tip. When tip moves over the asperity, it does not remain on the surface, but 
it mainly "flies" above the asperity and lands again on the surface, affecting the obtained values of friction coefficient. This is a big problem for measurements at nanoscale, since these kind of asperities could be macroparticles or defects embedded in the films and influence at a great extent the friction coefficient, giving "false" impressions for the tribological characteristics of the films. Although, the method described above, offers the tool to avoid these areas and measure at places that are defect-free, interpreting statistically more correct friction coefficient values of the films.

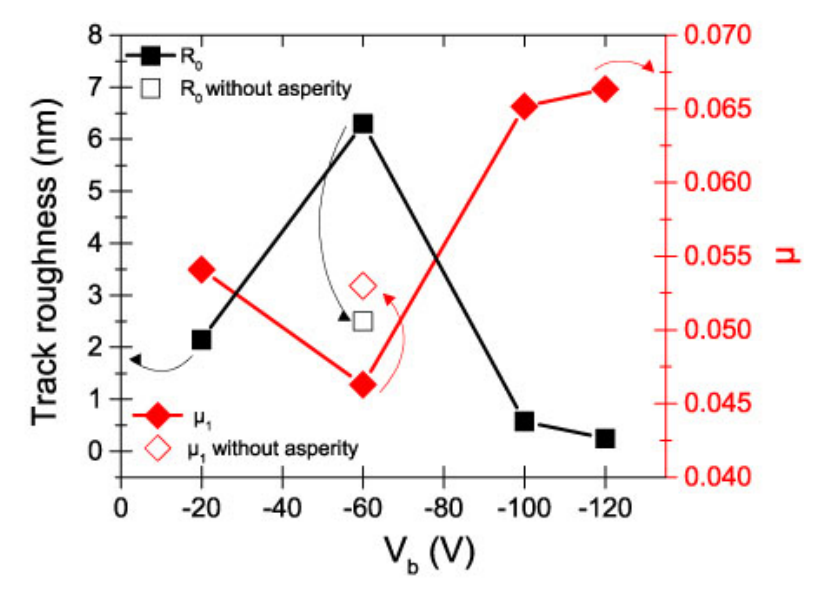

Figure 3.16: Initial track roughness (squares) and friction coefficient as obtained from the 1st cycle of the wear test as a function of $V_{b}$. The open square is the initial track roughness of the film grown at $V_{b}=60 \mathrm{~V}$ without included a large asperity detected on the track and the corresponding friction coefficient at the part of the track without the asperity.

${ }_{a}-C N_{x}$ thin films deposited with MFMS exhibited low to very low wear rates as is shown in Fig. 3.17.

It is noted that the films deposited with MFMS at $V_{b}=120 \mathrm{~V}$ showed negative wear, which accounts for a material volume that "grows" inside the wear track during the wear test. It is demonstrated in Paper II that this is explained by a surface modification; a phase transformation, possibly graphitization, often observed at the macroscale after friction and wear testing. This surface modification was possible to be observed in the $C N_{x}$ films of this study due to the low roughness of the track surface and the very low wear of this film. There are evidences where protuberances were observed at nanoscale, when very thin DLC films (between 0.5 and $2 \mathrm{~nm}$ ) were scratched [74]. This was ascribed to transfer of material from the Si substrate, 


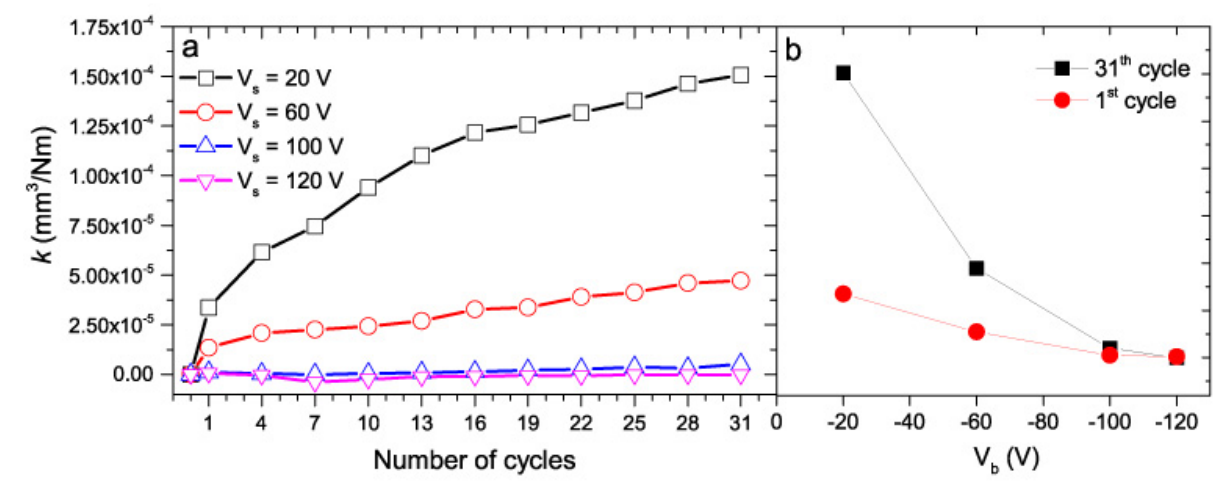

Figure 3.17: Wear coefficient of $C N_{x}$ thin films deposited by MFMS (triangles), HiPIMS (circles) and DCMS (squares) as a function of $V_{b}$.

which thereafter was oxidized, forming a thin layer of $\mathrm{SiO}_{2}$ on the surface above the $D L C$ film. This is not the case herein, since the thickness of our films is between 800 and $1100 \mathrm{~nm}$. 
Chapter 4

\section{Papers}




\section{Included papers}

Paper I: Bakoglidis K.D., Schmidt S., Garbrecht M., Ivanov G.I., Jensen J., Greczynski G., Hultman L. Low-temperature growth of low friction wear-resistant amorphous carbon nitride thin films by mid-frequency, high power impulse, and direct current magnetron sputtering (2015)

Submitted to Journal of Vacuum Science and Technology A

Paper II: Bakoglidis K.D., Broitman E., Schmidt S., Greczynski G., Hultman L. Nanotribological properties of wear-resistant amorphous carbon nitride thin films deposited by mid-frequency magnetron sputtering (2015)

In manuscript 


\section{Papers}

The articles associated with this thesis have been removed for copyright reasons. For more details about these see:

http://urn.kb.se/resolve?urn=urn:nbn:se:liu:diva-118349 


\section{Chapter 5}

\section{Conclusions and upcoming work}

\subsection{Conclusions}

The work included in this licentiate thesis is the first step to produce improved $C$-based thin films for bearing applications. The films must fulfill the specific mechanical and tribological properties of high density and high hardness, in order to withstand the demanding conditions of high load and long running time. An industrial deposition chamber was involved in order to ensure the industrial applicability and the potential commercialization of the synthetic methods. Three PVD magnetron sputtering techniques were explored and compared, namely MFMS, HiPIMS, and DCMS, in order to understand the potential of each of them, to grow $C N_{x}$ films with the desired structures and properties. $C N_{x}$ thin films were synthesized on steel AISI 52100 and Si(001) substrates. The results of the study regarding their structure, mechanical properties and tribological behavior are concluded below:

- Reactive magnetron sputtering of graphite targets in $N_{2} / A r$ atmosphere and at low temperatures $\left(T<150^{\circ} C\right)$, produced amorphous carbon nitride $a-C N_{x}$ thin films in either MFMS, HiPIMS or DCMS mode.

- $s p^{2}$-rich films were grown by all deposition techniques (Fig. 3.1), although an accurate estimation of the $s p^{3} / s p^{2}$ ratio in $C N_{x}$ films is not possible, using only the characterization techniques employed in this study (XPS).

- Substrate bias highly affects the structure and the mechanical properties of the $C N_{x}$ films deposited either with MFMS, HiPIMS or DCMS. Moreover the influence of substrate bias differs between the deposition techniques. 
- The morphology of the films is influenced by the applied substrate bias. A porous structure with columnar voids was developed when $20 \mathrm{~V}$ bias was used. Voids closed, porosity decreased and dense films formed at $V_{s} \geq 60 \mathrm{~V}, V_{s} \geq 100$ $V$, and $V_{s} \geq 120 V$ for MFMS, DCMS, and HiPIMS, respectively.

- $O$ content in the films decreased with increasing substrate bias, reflecting the decrease in films porosity.

- The $A r$ incorporation in the films increased with substrate bias for all deposition techniques. Moreover, different Ar uptake is observed for each technique,:

- The abundance of MFMS and DCMS processes in $A r^{+}$and the higher homogeneity of the films produced by these techniques result higher $\mathrm{Ar}$ intercalation than in films deposited by HiPIMS.

- The lower $A r^{+}$amount and the low duty cycles of the substrate bias of HiPIMS processes benefit low $A r$ incorporation rates in the films at all bias voltages.

- Denser $C N_{x}$ films are grown at higher substrate bias, due to the bombardment of the substrates with high energy ions.

- Substrate bias affects the residual stress of the $C N_{x}$ films. Stress increases linearly with increasing substrate bias in each deposition technique. Furthermore, the increase of residual stress of the $C N_{x}$ films is affected to different extent in each technique.

- Films produced by MFMS are affected to a higher extent and present the highest stress at each substrate bias. For these films, residual stress increases linearly to as high as $-4.2 \mathrm{GPa}$ at $V_{b}=120 \mathrm{~V}$

- Films produced by HiPIMS are affected to a less extent and present the lowest stress at each substrate bias. For these films, residual stress increases as high as $-1.2 \mathrm{GPa}$

- Films produced by DCMS are affected more than films produced by HiPIMS and less than films produced by MFMS, presenting an intermediate behaviour. For these films, residual stress increases up to to $-2.1 G P a$ at $V_{b}=120 \mathrm{~V}$ 
- MFMS produced harder films at each substrate bias than HiPIMS and DCMS. The hardness and density of the films grown by MFMS increases linearly with increasing bias voltage to as high as $\sim 25 G P a$ and $\sim 2.23 \mathrm{~g} / \mathrm{cm}^{3}$, respectively. HiPIMS produced softer films at each substrate bias, with the hardest film deposited at substrate bias of $120 \mathrm{~V}$ and hardness of $\sim 14 \mathrm{GPa}$.

- Low friction coefficients were recorded for all films and range between 0.05 and 0.07, when measured at the microscale. Friction coefficients did not show dependency on the substrate bias. They are generally influenced by the surface roughness of the tracks or the movements of the tip over the surface during the measurements.

- For films deposited by MFMS, friction coefficients were influenced by surface roughness in the tracks. Friction behaviour is governed by two lubrication mechanisms: a) debris particles at the first cycles and b) modifications on the surface (i.e., possible graphitization) after debris removal.

- The wear resistance of the $C N_{x}$ films increases with increasing substrate bias in each technique, due to the denser and harder structures obtained at higher substrate biases.

DCMS is confirmed as a technique with stable ionization conditions and in comparison to HiPIMS and MFMS, presents intermediate trends regarding the tribological and mechanical characteristics of the $C N_{x}$ films.

MFMS is revealed as a very robust technique for the production of low friction and wear resistant $C N_{x}$ films. The special cathode-anode configuration in MFMS processes provides with stable ionization conditions during the operation of graphite targets, which results to arc-free processes and beneficial surface characteristics. Furthermore, high ion energies during MFMS processes proved that hard and elastic ${ }_{a}-C N_{x}$ films can be synthesized with this technique. However, the high induced film stress causes film delamination following the deposition, and makes the use of an adhesion enhancement technique necessary, if MFMS is used for $C N_{x}$ film depositions.

On the other hand, if high hardness is not requisite for a specific application, HiPIMS produces $C N_{x}$ films with very low stress, which poses as a basic requirement for better adhesion of $C N_{x}$ coatings deposited on steel substrates. In this case, an adhesion pre-treatment prior to deposition may be better exploited, since the stress 
level is lower, offering benefits in the overall performance of the coating. Coatings grown by HiPIMS also possess low friction, although their wear resistance is lower than films grown by MFMS and DCMS. However, the friction coefficient values and the wear rates of the films grown by HiPIMS are not prohibitive for use in applications, depending on the requirements and the work load of each application. On the disadvantages side, the severe arcing of graphite targets in HiPIMS processes leads to a film with defects on the surface or even embedded in the film structure, such as macroparticles, which may influence the surface characteristics of the films and friction to a higher extent.

\subsection{Upcoming work}

After achieving appropriate low friction and wear resistant $a-C N_{x}$ thin films, attention should be put on the improvement of adhesion between the $a-C N_{x}$ films and steel substrates, either flat disks or cylindrical rollers. Adhesion improvements can be directed by a pre-treatment step for the substrates prior to the depositions of $a$ $C N_{x}$, using metal ion etching or combined gas ion-metal ion etching of the substrate surface. This step is best conducted with HiPIMS, since it has been proven that high energy metal ions in HiPIMS promote film/substrate adhesion [17] [18]. The pre-treatment procedure should be optimized for the AISI 52100 bearing steel. For this reason, a special HiPIMS pre-treatment should be investigated, using different metals in HiPIMS mode, in order to find the extent to which each pre-treatment affects the adhesion of $C N_{x}$ films on bearing steel. Thus, the respective consequences of each pre-treatment to the lifetime of coatings and rollers will also be studied. It is important to understand the mechanisms of adhesion. The high energy ion etching promotes a mechanism referred to as "stitching" [75], which depends on the chemical bonding on the substrate surface and consequently on the involved materials. The analysis of the $C N_{x}$ film/AISI 52100 steel substrate interface will be conducted using TEM imaging techniques together with electron dispersive X-ray spectroscopy (EDX) or electron energy loss spectroscopy (EELS), which will give insights to the stitching mechanism for each metal pre-treatment, while XPS and time-of-flight secondary ion mass spectrometry (ToF-SIMS) will reveal the different chemical bonding configurations at the interface.

Another important step is the deposition of $a-C N_{x}$ coatings on rollers and their testing against steel surfaces, under real rolling and sliding conditions, simulating 
the actual roller bearing operation. This will give a sense of how the $a-C N_{x}$ coatings operate under high loads and if, and how, they change the properties of the roller surface. Moreover, analysis of the wear tracks of rollers and rings is judged of critical importance, in order to reveal possible failure mechanisms of $a-C N_{x}$ coated and uncoated steel rollers. I will try to investigate and understand the friction and wear as well as the lubrication mechanisms that govern the operation of $C N_{x}$ coated rollers. A graphitization process is common for $C$-based films when tested with tribological tests and this mechanism is assumed to be responsible for the low friction provided by such films [76], [77]. The wear track analysis will be conducted using TEM/EDX (or EELS) and SEM imaging techniques. The comparison of the initial and final roughness of the counterparts is also important for the evaluation of the tribological performance of coated rollers and will be conducted using surface profilometry and scanning probe microscopy (SPM). The mechanisms of lubrication for the coated rollers will be analyzed using Raman spectroscopy and XPS, which will reveal the chemical environment and bonding configuration of the $C N_{x}$ films, before and after the wear tests.

Possible improvements of the mechanical and tribological properties of $a-C N_{x}$ and $a-C$ thin films will also be investigated through structural changes induced by doping of the films with low doses of metal ions, exploiting the potential of the magnetron sputtering techniques reported herein. Recently, the doping of graphitelike carbon coatings with $C r$ has been reported with beneficial effects on friction and wear of the coatings, where the lubrication of the contact in a pin-on-disk test was improved due to a $\mathrm{CrCl}_{3}$ tribolayer, formed by chemical reaction presence of lubricant [78]. The effort will be to improve the mechanical and tribological properties of the $a-C N_{x}$ films, with the addition of a metal and to explore the potential of further optimization under lubricated conditions. Thus, the reactions of doped- $C N_{x}$ films and coated rollers in the presence of a lubricant are also of high importance to understand. 


\section{Chapter 6}

\section{Bibliography}

[1] K Holmberg and A Matthews. Coatings Tribology: Properties, Mechanisms, Techniques, and Applications in Surface Engineering, Tribology and Interface Engineering Series, 56. Elsevier, 2009.

[2] H. K D H Bhadeshia. Steels for bearings. Progress in Materials Science, 57(2):268-435, 2012.

[3] Andrey A. Voevodin and J. S. Zabinski. Nanocomposite and nanostructured tribological materials for space applications. Composites Science and Technology, 65(5 SPEC. ISS.):741-748, 2005.

[4] B. O. Johansson. Growth and properties of single crystal TiN films deposited by reactive magnetron sputtering. Journal of Vacuum Science 83 Technology A: Vacuum, Surfaces, and Films, 3(2):303, 1985.

[5] Bharat Bhushan. Springer Handbook of Nanotechnology. Springer Science \& Business Media, 2010.

[6] T. W. Scharf, R. D. Ott, D. Yang, and J. A. Barnard. Structural and tribological characterization of protective amorphous diamond-like carbon and amorphous $C N_{x}$ overcoats for next generation hard disks. Journal of Applied Physics, 85(1999):3142, 1999.

[7] J Robertson. Diamond-like amorphous carbon. Materials Science and Engineering: R: Reports, 37:129-281, 2002. 
[8] C. A. Charitidis. Nanomechanical and nanotribological properties of carbonbased thin films: A review. International Journal of Refractory Metals and Hard Materials, 28(1):51-70, 2010.

[9] A. Erdemir, O.L. Eryilmaz, and S.H. Kim. Effect of tribochemistry on lubricity of DLC films in hydrogen. Surface and Coatings Technology, 257:241-246, 2014.

[10] Andrey A. Voevodin, J. G. Jones, T. C. Back, J. S. Zabinski, V. E. Strel'nitzki, and I. I. Aksenov. Comparative study of wear-resistant DLC and fullerene-like CNx coatings produced by pulsed laser and filtered cathodic arc depositions. Surface and Coatings Technology, 197:116-125, 2005.

[11] J. J. Rha, S. C. Kwon, J. R. Cho, S. Yim, and N. Saka. Creation of ultra-low friction and wear surfaces for micro-devices using carbon films. Wear, 259:765$770,2005$.

[12] Lars Hultman, Jörg Neidhardt, Niklas Hellgren, Hans Sjöström, and Jan-Eric Sundgren. Fullerene-like Carbon Nitride: A Resilient Coating Material. MRS Bulletin, 28(March):194-202, 2003.

[13] Wolfgang Tillmann, Evelina Vogli, and Fabian Hoffmann. Wear-resistant and low-friction diamond-like-carbon (DLC)-layers for industrial tribological applications under humid conditions. Surface and Coatings Technology, 204(67):1040-1045, 2009.

[14] Andrea Carlo Ferrari. Diamond-like carbon for magnetic storage disks. Surface and Coatings Technology, 180-181:190-206, 2004.

[15] Arto Koistinen, Seppo S. Santavirta, Heikki Kröger, and Reijo Lappalainen. Effect of bone mineral density and amorphous diamond coatings on insertion torque of bone screws. Biomaterials, 26:5687-5694, 2005.

[16] Wolfgang Tillmann, Evelina Vogli, and Siavash Momeni. Effect of Plasma Treatment on Adhesion of DLC Layers to Steels. Materials Science Forum, 638-642:812-817, 2010.

[17] M. Lattemann, a. P. Ehiasarian, J. Bohlmark, P. A O Persson, and U. Helmersson. Investigation of high power impulse magnetron sputtering pretreated interfaces for adhesion enhancement of hard coatings on steel. Surface and Coatings Technology, 200:6495-6499, 2006. 
[18] E. Broitman, Zs Czigány, G. Greczynski, J. Böhlmark, R. Cremer, and L. Hultman. Industrial-scale deposition of highly adherent $\mathrm{CNx}$ films on steel substrates. Surface and Coatings Technology, 204(21-22):3349-3357, 2010.

[19] Chun-Chin Chen and Franklin Chau-Nan Hong. Interfacial studies for improving the adhesion of diamond-like carbon films on steel. Applied Surface Science, 243:296-303, 2005.

[20] J. Neidhardt, H. Högberg, and L. Hultman. Cryogenic deposition of carbon nitride thin solid films by reactive magnetron sputtering; Suppression of the chemical desorption processes. Thin Solid Films, 478:34-41, 2005.

[21] S. Schmidt, Zs Czigány, G. Greczynski, J. Jensen, and L. Hultman. Ion mass spectrometry investigations of the discharge during reactive high power pulsed and direct current magnetron sputtering of carbon in $\mathrm{Ar}$ and $\mathrm{Ar} / N_{2}$. Journal of Applied Physics, 112(2012), 2012.

[22] J. Neidhardt, L. Hultman, E. Broitman, T. W. Scharf, and I. L. Singer. Structural, mechanical and tribological behavior of fullerene-like and amorphous carbon nitride coatings. Diamond and Related Materials, 13:1882-1888, 2004.

[23] HZ Schmellenmeier. Carbon layers with diamond structure. Z Phys Chem DDR, 1956.

[24] Sol Aisenberg and Ronald Chabot. Ion-beam deposition of thin films of diamondlike carbon. Journal of Applied Physics, 42(7):2953-2958, 1971.

[25] Y. Lifshitz. Diamond-like carbon - present status. Diamond and Related Materials, 8(8-9):1659-1676, 1999.

[26] G. K. Gueorguiev, J. Neidhardt, S. Stafström, and L. Hultman. First-principles calculations on the curvature evolution and cross-linkage in carbon nitride. Chemical Physics Letters, 410:228-234, 2005.

[27] A Y Liu and M L Cohen. Prediction of new low compressibility solids. Science (New York, N.Y.), 245:841-842, 1989.

[28] A.Y. Liu and M.L. Cohen. Structural properties and electronic structure of lowcompressibility materials; $\beta-S i_{3} N_{4}$ and hypothetical $\beta-C_{3} N_{4}$. Physical Review $B, 41(15): 055502,1990$. 
[29] Hyunchae Cynn, John E Klepeis, Choong-Shik Yoo, and David a Young. Osmium has the lowest experimentally determined compressibility. Physical review letters, 88(13):135701, 2002.

[30] S. E. Rodil and S. Muhl. Bonding in amorphous carbon nitride. Diamond and Related Materials, 13:1521-1531, 2004.

[31] A. C. Ferrari, S. E. Rodil, and J. Robertson. Resonant Raman spectra of amorphous carbon nitrides: The G peak dispersion. Diamond and Related Materials, 12(02):905-910, 2003.

[32] M. Gioti, S. Logothetidis, P. Patsalas, A. Laskarakis, Y. Panayiotatos, and V. Kechagias. Magnetron sputtered carbon nitride: Composition and chemical bonding of as-grown and post-annealed films studied with real-time and in situ diagnostic techniques. Surface and Coatings Technology, 125:289-294, 2000.

[33] H. Sjöström and H Sjöström. Structural and mechanical properties of carbon nitride $C N_{x}(0.2 \leq x \leq 0.35)$ films. Journal of Vacuum Science 85 Technology A: Vacuum, Surfaces, and Films, 14(1996):56, 1996.

[34] J. Neidhardt, Zs. Czigany, I. F. Brunell, and L. Hultman. Growth of fullerenelike carbon nitride thin solid films by reactive magnetron sputtering; Role of low-energy ion irradiation in determining microstructure and mechanical properties. Journal of Applied Physics, 93(2003):3002-3015, 2003.

[35] H. Sjöström, S. Stafström, M. Boman, and J.-E. Sundgren. Superhard and Elastic Carbon Nitride Thin Films Having Fullerenelike Microstructure. Phys. Rev. Lett., 75(7):1336, 1995.

[36] Niklas Hellgren, Mats Johansson, Esteban Broitman, Lars Hultman, and JanEric Sundgren. Role of nitrogen in the formation of hard and elastic $C N_{x}$ thin films by reactive magnetron sputtering. Physical Review B, 59(7):5162-5169, 1999.

[37] Niklas Hellgren, Mats P. Johansson, Esteban Broitman, Per Sandström, Lars Hultman, Jan-eric Eric Sundgren, Niklas Hellgren U, Mats P. Johansson, Esteban Broitman, Per Sandstrom, Lars Hultman, and Jan-eric Eric Sundgren. Effect of chemical sputtering on the growth and structural evolution of magnetron sputtered $C N_{x}$ thin films. Thin Solid Films, 382:146-152, 2001. 
[38] Susann Schmidt, Zsolt Czigány, Grzegorz Greczynski, Jens Jensen, and Lars Hultman. Influence of inert gases on the reactive high power pulsed magnetron sputtering process of carbon-nitride thin films. Journal of Vacuum Science $\mathscr{E}$ Technology A: Vacuum, Surfaces, and Films, 31:011503, 2013.

[39] G. K. Gueorguiev, E. Broitman, A. Furlan, S. Stafström, and L. Hultman. Dangling bond energetics in carbon nitride and phosphorus carbide thin films with fullerene-like and amorphous structure. Chemical Physics Letters, 482(13):110-113, 2009.

[40] C. Charitidis and S. Logothetidis. Nanomechanical and nanotribological properties of carbon based films. Thin Solid Films, 482:120-125, 2005.

[41] A. Champi, F. C. Marques, and F. L. Freire. Effect of the bias voltage on the structure of carbon nitride films. Diamond and Related Materials, 13:1538$1542,2004$.

[42] E. Broitman, G. K. Gueorguiev, A. Furlan, N. T. Son, a. J. Gellman, S. Stafström, and L. Hultman. Water adsorption on fullerene-like carbon nitride overcoats. Thin Solid Films, 517(3):1106-1110, 2008.

[43] Niklas Hellgren, Karol Macák, Esteban Broitman, Mats P. Johansson, Lars Hultman, and Jan-eric Sundgren. Influence of plasma parameters on the growth and properties of magnetron sputtered $C N_{x}$ thin films. Journal of Applied Physics, 524(2000):524, 2003.

[44] E Riedo. Structural properties and surface morphology of laser-deposited amorphous carbon and carbon nitride films. Surface and Coatings Technology, 125:124-128, 2000.

[45] Mark D. Tucker, Zsolt Czigány, Esteban Broitman, Lars-Åke Näslund, Lars Hultman, and Johanna Rosen. Filtered pulsed cathodic arc deposition of fullerene-like carbon and carbon nitride films. Journal of Applied Physics, 115(May):144312, 2014.

[46] R. Gago, J. Neidhardt, M. Vinnichenko, U. Kreissig, Zs Czigány, a. Kolitsch, L. Hultman, and W. Möller. Synthesis of carbon nitride thin films by lowenergy ion beam assisted evaporation: On the mechanisms for fullerene-like microstructure formation. Thin Solid Films, 483:89-94, 2005. 
[47] B. Kleinsorge, A. C. Ferrari, J. Robertson, and W. I. Milne. Influence of nitrogen and temperature on the deposition of tetrahedrally bonded amorphous carbon. Journal of Applied Physics, 88(2000):1149, 2000.

[48] M. Ohring. Materials Science of Thin Films. Academic Press, 2001.

[49] P. Hammer. Synthesis of carbon nitride films at low temperatures. Journal of Vacuum Science \& Technology A: Vacuum, Surfaces, and Films, 15(1997):107, 1997.

[50] R. Kaltofen. Plasma diagnostic studies to the carbon nitride film deposition by reactive r.f. magnetron sputtering. Thin Solid Films, 290-291:112-119, 1996.

[51] I. K. Fetisov, A. A. Filippov, G. V. Khodachenko, D. V. Mozgrin, and A. A. Pisarev. Impulse irradiation plasma technology for film deposition. Vacuum, 53(1-2):133-136, 1999.

[52] Vladimir Kouznetsov, Karol Macák, Jochen M. Schneider, Ulf Helmersson, and Ivan Petrov. A novel pulsed magnetron sputter technique utilizing very high target power densities. Surface and Coatings Technology, 122(2-3):290-293, 1999.

[53] K. Sarakinos, J. Alami, and S. Konstantinidis. High power pulsed magnetron sputtering: A review on scientific and engineering state of the art. Surface and Coatings Technology, 204(11):1661-1684, 2010.

[54] B.M. DeKoven, P.R. Ward, R.E. Weiss, D.J. Christie, R.A. Scholl, F. Tomasel, and A. Anders. Carbon thin film deposition using high power pulsed magnetronsputtering. Society of Vacuum Coaters 46th Annual Technical Conference Proceedings, pages 158-165, 2003.

[55] Ante Hecimovic and Arutiun P. Ehiasarian. Temporal evolution of the ion fluxes for various elements in HIPIMS plasma discharge. IEEE Transactions on Plasma Science, 39(4 PART 2):1154-1164, 2011.

[56] A. Laskarakis, S. Logothetidis, C. Charitidis, M. Gioti, and Y. Panayiotatos. A study on the bonding structure and mechanical properties of magnetron sputtered $C N_{x}$ thin films. Diamond and Related Materials, pages 1179-1184, 2001. 
[57] I. Petrov, P. B. Barna, L. Hultman, and J. E. Greene. Microstructural evolution during film growth. Journal of Vacuum Science \& Technology A: Vacuum, Surfaces, and Films, 21:S117, 2003.

[58] M. Knoll and E. Ruska. Das elektronenmikroskop. Zeitschrift für Physik, 1932.

[59] L. De Broglie. Sur la fréquence propre de l'électron. Compt. Rend. Acad. Sci, 1925.

[60] D.B. Williams and C.B. Carter. Transmission Electron Microscopy. Springer US, Boston, MA, 2009.

[61] J. Neidhardt, L. Hultman, and Zs. Czigány. Correlated high resolution transmission electron microscopy and X-ray photoelectron spectroscopy studies of structured $C N_{x}(0<x<0.25)$ thin solid films. Carbon, 42:2729-2734, 2004.

[62] Zsolt Czigány and Lars Hultman. Interpretation of electron diffraction patterns from amorphous and fullerene-like carbon allotropes. Ultramicroscopy, 110:815$819,2010$.

[63] Zs Czigány, I. F. Brunell, J. Neidhardt, L. Hultman, and K. Suenaga. Growth of fullerene-like carbon nitride thin solid films consisting of cross-linked nanoonions. Applied Physics Letters, 79(2001):2639-2641, 2001.

[64] Zs. Czigány, J. Neidhardt, I. F. Brunell, and L. Hultman. Imaging of fullerenelike structures in $C N_{x}$ thin films by electron microscopy; Sample preparation artefacts due to ion-beam milling. Ultramicroscopy, 94:163-173, 2003.

[65] R. M. Langford and A. K. Petford-Long. Preparation of transmission electron microscopy cross-section specimens using focused ion beam milling. Journal of Vacuum Science \& Technology A: Vacuum, Surfaces, and Films, 19(May 2014):2186, 2001.

[66] Niklas Hellgren, Jinghua Guo, Yi Luo, Conny Såthe, Akane Agui, Stepan Kashtanov, Joseph Nordgren, Hans Ågren, and Jan Eric Sundgren. Electronic structure of carbon nitride thin films studied by X-ray spectroscopy techniques. Thin Solid Films, 471:19-34, 2005.

[67] W.T. Zheng, K.Z. Xing, N. Hellgren, M. Lögdlund, Å. Johansson, U. Gelivs, W.R. Salaneck, and J.-E. Sundgren. Nitrogen 1s electron binding energy assign- 
ment in carbon nitride thin films with different structures. Journal of Electron Spectroscopy and Related Phenomena, 87:45-49, 1997.

[68] Javier Díaz, Guido Paolicelli, Salvador Ferrer, and Fabio Comin. Separation of the $s p^{3}$ and $s p^{2}$ components in the C1s photoemission spectra of amorphous carbon films. Physical Review B, 54(11):8064-8069, 1996.

[69] Rainer Haerle, Elisa Riedo, Alfredo Pasquarello, and Alfonso Baldereschi. $s p^{2} / s p^{3}$ hybridization ratio in amorphous carbon from C1s core-level shifts: $\mathrm{X}$-ray photoelectron spectroscopy and first-principles calculation. Physical Review B, 65:32-37, 2001.

[70] M.A. Hopcroft, W.D. Nix, and T.W. Kenny. What is the Young's Modulus of Silicon? Journal of microelectromechanical systems, 19(2):229-238, 2010.

[71] H. Windischmann. Intrinsic stress in sputter-deposited thin films. Critical Reviews in Solid State and Materials Sciences, 17(6):547-596, 1992.

[72] W.C. Oliver and G.M. Pharr. An improved technique for determining hardness and elastic modulus using load and displacement sensing indentation experiments. Journal of Materials Research, 7(1):1564-1583, 1992.

[73] J. F. Archard and W. Hirst. The Wear of Metals under Unlubricated Conditions. Proceedings of the Royal Society A: Mathematical, Physical and Engineering Sciences, 236(August):397-410, 1956.

[74] Shojiro Miyake and Shohei Yamazaki. Evaluation of protuberance and groove formation in extremely thin DLC films on Si substrates due to diamond tip sliding by atomic force microscopy. Wear, 318(1-2):135-144, 2014.

[75] J. J. Cuomo, S. M. Rossnagel, H. R. Haufman, and Ranga Komanduri. Handbook of Ion Beam Processing Technology: Principles, Deposition, Film Modification, and Synthesis, 1990.

[76] Kenneth Holmberg, Allan Matthews, and Helena Ronkainen. Coatings tribology - contact mechanisms and surface design. Tribology International, 31(98):107-120, 1998.

[77] Dong Wook Kim and Kyung Woong Kim. Effects of sliding velocity and normal load on friction and wear characteristics of multi-layered diamond-like carbon (DLC) coating prepared by reactive sputtering. Wear, 297(1-2):722-730, 2013. 
[78] Paranjayee Mandal, Arutiun P. Ehiasarian, and Papken Eh. Hovsepian. Lubricated sliding wear mechanism of chromium-doped graphite-like carbon coating. Tribology International, 77:186-195, 2014. 